\title{
Hermite-Hadamard type inequalities for $F$-convex function involving fractional integrals
}

\author{
Pshtiwan Othman Mohammed ${ }^{1 *}$ (D) and Mehmet Zeki Sarikaya ${ }^{2}$
}

"Correspondence:
pshtiwansangawi@gmail.com
${ }^{1}$ Department of Mathematics,
College of Education, University of
Sulaimani, Sulaimani, Iraq
Full list of author information is
available at the end of the article

available at the end of the article

\begin{abstract}
In this study, the family $F$ and $F$-convex function are given with its properties. In view of this, we establish some new inequalities of Hermite-Hadamard type for differentiable function. Moreover, we establish some trapezoid type inequalities for functions whose second derivatives in absolute values are $F$-convex. We also show that through the notion of F-convex we can find some new Hermite-Hadamard type and trapezoid type inequalities for the Riemann-Liouville fractional integrals and classical integrals.
\end{abstract}

MSC: 26A51; 26D15; 35A23

Keywords: $F$-convex; $\lambda_{\varphi}$-preinvex; Integral inequalities

\section{Introduction}

A function $f: I \subseteq \mathbb{R} \rightarrow \mathbb{R}$ is said to be convex on the interval $I$, if for all $x, y \in I$ and $t \in(0,1)$ it satisfies the following inequality:

$$
f(t x+(1-t) y) \leq t f(x)+(1-t) f(y)
$$

Convex functions play an important role in the field of integral inequalities. For convex functions, many equalities and inequalities have been established, but one of the most important ones is the Hermite-Hadamard' integral inequality, which is defined as follows [1]:

Let $f: I \subseteq \mathbb{R} \rightarrow \mathbb{R}$ be a convex function with $a<b$ and $a, b \in I$. Then the HermiteHadamard inequality is given by

$$
f\left(\frac{a+b}{2}\right) \leq \frac{1}{b-a} \int_{a}^{b} f(x) d x \leq \frac{f(a)+f(b)}{2} .
$$

In recent years, a number of mathematicians have devoted their efforts to generalizing, refining, counterparting, and extending the Hermite-Hadamard inequality (2) for different classes of convex functions and mappings. The Hermite-Hadamard inequality (2) is established for the classical integral, fractional integrals, conformable fractional integrals and most recently for generalized fractional integrals; see for details and applications [2-8] and the references therein.

(c) The Author(s) 2018. This article is distributed under the terms of the Creative Commons Attribution 4.0 International License (http://creativecommons.org/licenses/by/4.0/), which permits unrestricted use, distribution, and reproduction in any medium, provided you give appropriate credit to the original author(s) and the source, provide a link to the Creative Commons license, and indicate if changes were made. 
The concepts of classical convex functions have been extended and generalized in several directions, such as quasi-convex [9], pseudo-convex [10], $M T$-convex [11] strongly convex [12], $\epsilon$-convex [13], $s$-convex [14], $h$-convex [15], and $\lambda_{\varphi}$-preinvex [16]. Recently, Samet [17] has defined a new concept of convexity that depends on a certain function satisfying some axioms, generalizing different types of convexity, including $\epsilon$-convex functions, $\alpha$-convex functions, $h$-convex functions, and so on, as stated in the next section.

\section{Review of the family of $\mathcal{F}$}

We address the family of $\mathcal{F}$ of mappings $F: \mathbb{R} \times \mathbb{R} \times \mathbb{R} \times[0,1] \rightarrow \mathbb{R}$ satisfying the following axioms:

(A1) If $u_{i} \in L^{1}(0,1), i=1,2,3$, then, for every $\lambda \in[0,1]$, we have

$$
\int_{0}^{1} F\left(u_{1}(t), u_{2}(t), u_{3}(t), \lambda\right) d t=F\left(\int_{0}^{1} u_{1}(t) d t, \int_{0}^{1} u_{2}(t) d t, \int_{0}^{1} u_{3}(t) d t, \lambda\right) .
$$

(A2) For every $u \in L^{1}(0,1), w \in L^{\infty}(0,1)$ and $\left(z_{1}, z_{2}\right) \in \mathbb{R}^{2}$, we have

$$
\int_{0}^{1} F\left(w(t) u(t), w(t) z_{1}, w(t) z_{2}, t\right) d t=T_{F, w}\left(\int_{0}^{1} w(t) u(t) d t, z_{1}, z_{2}\right)
$$

where $T_{F, w}: \mathbb{R} \times \mathbb{R} \times \mathbb{R} \rightarrow \mathbb{R}$ is a function that depends on $(F, w)$, and it is nondecreasing with respect to the first variable.

(A3) For any $\left(w, u_{1}, u_{2}, u_{3}\right) \in \mathbb{R}^{4}, u_{4} \in[0,1]$, we have

$$
w F\left(u_{1}, u_{2}, u_{3}, u_{4}\right)=F\left(w u_{1}, w u_{2}, w u_{3}, u_{4}\right)+L_{w},
$$

where $L_{w} \in \mathbb{R}$ is a constant that depends only on $w$.

Definition 2.1 Let $f:[a, b] \rightarrow \mathbb{R},(a, b) \in \mathbb{R}^{2}, a<b$, be a given function. We say that $f$ is a convex function with respect to some $F \in \mathcal{F}$ (or $F$-convex function) iff

$$
F(f(t x+(1-t) y), f(x), f(y), t) \leq 0, \quad(x, y, t) \in[a, b] \times[a, b] \times[0,1] .
$$

Remark 1 Suppose that $(a, b) \in \mathbb{R}^{2}$ with $a<b$.

(i) Let $f:[a, b] \rightarrow \mathbb{R}$ be an $\varepsilon$-convex function, that is [18],

$$
f(t x+(1-t) y) \leq t f(x)+(1-t) f(y), \quad(x, y, t) \in[a, b] \times[a, b] \times[0,1] .
$$

Define the functions $F: \mathbb{R} \times \mathbb{R} \times \mathbb{R} \times[0,1] \rightarrow \mathbb{R}$ by

$$
F\left(u_{1}, u_{2}, u_{3}, u_{4}\right)=u_{1}-u_{4} u_{2}-\left(1-u_{4}\right) u_{3}-\varepsilon
$$

and $T_{F, w}: \mathbb{R} \times \mathbb{R} \times \mathbb{R} \times[0,1] \rightarrow \mathbb{R}$ by

$$
T_{F, w}\left(u_{1}, u_{2}, u_{3}\right)=u_{1}-\left(\int_{0}^{1} t w(t) d t\right) u_{2}-\left(\int_{0}^{1}(1-t) w(t) d t\right) u_{3}-\varepsilon .
$$


For

$$
L_{w}=(1-w) \varepsilon
$$

it will be seen that $F \in \mathcal{F}$ and

$$
F(f(t x+(1-t) y), f(x), f(y), t)=f(t x+(1-t) y)-t f(x)-(1-t) f(y)-\varepsilon \leq 0,
$$

that is, $f$ is an $F$-convex function. Particularly, taking $\varepsilon=0$ we show that if $f$ is a convex function then $f$ is an $F$-convex function with respect to $F$ defined above.

(ii) Let $f:[a, b] \rightarrow \mathbb{R}$ be $\lambda_{\varphi}$-preinvex function according to $\varphi$ and bifunction $\eta$, $0 \leq \varphi \leq \frac{\pi}{2}, \lambda \in\left(0, \frac{1}{2}\right]$, that is $[16]$

$$
\begin{aligned}
f(u & \left.+t e^{i \varphi} \eta(v, u)\right) \\
& \leq \frac{\sqrt{t}}{2 \sqrt{1-t}} f(v)+\frac{(1-\lambda) \sqrt{1-t}}{2 \lambda \sqrt{t}} f(u), \quad(u, v, t) \in[a, b] \times[a, b] \times(0,1) .
\end{aligned}
$$

Define the functions $F: \mathbb{R} \times \mathbb{R} \times \mathbb{R} \times[0,1] \rightarrow \mathbb{R}$ by

$$
F\left(u_{1}, u_{2}, u_{3}, u_{4}\right)=u_{1}-\frac{\sqrt{u_{4}}}{2 \sqrt{1-u_{4}}} u_{3}-\frac{(1-\lambda) \sqrt{1-u_{4}}}{2 \lambda \sqrt{u_{4}}} u_{2}
$$

and $T_{F, w}: \mathbb{R} \times \mathbb{R} \times \mathbb{R} \times[0,1] \rightarrow \mathbb{R}$ by

$$
\begin{aligned}
T_{F, w}\left(u_{1}, u_{2}, u_{3}\right)= & u_{1}-\left(\int_{0}^{1} \frac{\sqrt{t}}{2 \sqrt{1-t}} w(t) d t\right) u_{3} \\
& -\frac{1-\lambda}{\lambda}\left(\int_{0}^{1} \frac{\sqrt{1-t}}{2 \sqrt{t}} w(t) d t\right) u_{2} .
\end{aligned}
$$

For $L_{w}=0$, it will be seen that $F \in \mathcal{F}$ and

$$
\begin{aligned}
& F\left(f\left(u+t e^{i \varphi} \eta(v, u)\right), f(u), f(v), t\right) \\
& \quad=f\left(u+t e^{i \varphi} \eta(v, u)\right)-\frac{\sqrt{t}}{2 \sqrt{1-t}} f(v)-\frac{(1-\lambda) \sqrt{1-t}}{2 \lambda \sqrt{t}} f(u)-\varepsilon \leq 0,
\end{aligned}
$$

that is $f$ is an $F$-convex function.

(iii) Let $h: I \rightarrow \mathbb{R}$ be a given function which is not identical to 0 , where $I$ is an interval in $\mathbb{R}$ such that $(0,1) \subseteq I$. Let $f:[a, b] \rightarrow[0, \infty)$ be an $h$-convex function, that is,

$$
f(t x+(1-t) y) \leq h(t) f(x)+\frac{1-\lambda}{\lambda} h(1-t) f(y), \quad(x, y, t) \in[a, b] \times[a, b] \times[0,1] .
$$

Define the functions $F: \mathbb{R} \times \mathbb{R} \times \mathbb{R} \times[0,1] \rightarrow \mathbb{R}$ by

$$
F\left(u_{1}, u_{2}, u_{3}, u_{4}\right)=u_{1}-h\left(u_{4}\right) u_{3}-\frac{1-\lambda}{\lambda} h\left(1-u_{4}\right) u_{2}
$$


and $T_{F, w}: \mathbb{R} \times \mathbb{R} \times \mathbb{R} \times[0,1] \rightarrow \mathbb{R}$ by

$$
T_{F, w}\left(u_{1}, u_{2}, u_{3}\right)=u_{1}-\left(\int_{0}^{1} h(t) w(t) d t\right) u_{3}-\frac{1-\lambda}{\lambda}\left(\int_{0}^{1} h(1-t) w(t) d t\right) u_{2} .
$$

For $L_{w}=(1-w) \varepsilon$, it will be seen that $F \in \mathcal{F}$ and

$$
\begin{aligned}
& F(f(t x+(1-t) y), f(x), f(y), t) \\
& \quad=f(t x+(1-t) y)-h(t) f(x)-\frac{1-\lambda}{\lambda} h(1-t) f(y)-\varepsilon \leq 0,
\end{aligned}
$$

that is $f$ is an $F$-convex function.

Recently Samet [17] established some integral inequalities of Hermite-Hadamard type via $F$-convex functions.

Theorem 1 ([17, Theorem 3.1]) Let $f:[a, b] \rightarrow \mathbb{R},(a, b) \in \mathbb{R}^{2}, a<b$, be an $F$-convex function, for some $F \in \mathcal{F}$. Suppose that $F \in L^{1}(a, b)$. Then

$$
\begin{aligned}
& F\left(f\left(\frac{a+b}{2}\right), \frac{1}{b-a} \int_{a}^{b} f(x) d x, \frac{1}{2}\right) \leq 0, \\
& T_{F, 1}\left(\frac{1}{b-a} \int_{a}^{b} f(x) d x, f(a), f(b)\right) \leq 0 .
\end{aligned}
$$

Theorem 2 ([17, Theorem 3.4]) Let $f: I^{o} \subseteq \mathbb{R} \rightarrow \mathbb{R}$ be a differentiable mapping on $I^{o}$, $(a, b) \in I^{o} \times I^{o}, a<b$. Suppose that

(i) $\left|f^{\prime}\right|$ is $F$-convex on $[a, b]$, for some $F \in \mathcal{F}$;

(ii) the function $t \in(0,1) \rightarrow L_{w(t)}$ belongs to $L^{1}(a, b)$, where $w(t)=|1-2 t|$.

Then

$$
T_{F, w}\left(\frac{2}{b-a}\left|\frac{f(a)+f(b)}{2}-\frac{1}{b-a} \int_{a}^{b} f(x) d x\right|,\left|f^{\prime}(a)\right|,\left|f^{\prime}(b)\right|\right)+\int_{0}^{1} L_{w(t)} d t \leq 0 .
$$

Theorem 3 ([17, Theorem 3.5]) Let $f: I^{o} \subseteq \mathbb{R} \rightarrow \mathbb{R}$ be a differentiable mapping on $I^{o}$, $(a, b) \in I^{o} \times I^{o}, a<b$ and let $p>1$. Suppose that $\left|f^{\prime}\right|^{p /(p-1)}$ is F-convex on $[a, b]$, for some $F \in \mathcal{F}$ and $F \in L^{p /(p-1)}(a, b)$. Then

$$
T_{F, 1}\left(A(p, f),\left|f^{\prime}(a)\right|^{\frac{p}{p-1}},\left|f^{\prime}(b)\right|^{\frac{p}{p-1}}\right) \leq 0,
$$

where

$$
A(p, f)=\left(\frac{2}{b-a}\right)^{\frac{p}{p-1}}(p+1)^{\frac{1}{p-1}}\left|\frac{f(a)+f(b)}{2}-\frac{1}{b-a} \int_{a}^{b} f(x) d x\right|^{\frac{p}{p-1}} .
$$

As consequences of the above theorems, the author obtained some integral inequalities for $\varepsilon$-convexity, $\alpha$-convexity, and $h$-convexity. 
Theorem 4 ([17, Corollary 4.3]) Let $f: I^{o} \subseteq \mathbb{R} \rightarrow \mathbb{R}$ be a differentiable mapping on $I^{o}$, $(a, b) \in I^{o} \times I^{o}, a<b$. Suppose that the function $\left|f^{\prime}\right|$ is $\varepsilon$-convex on $[a, b], \varepsilon \geq 0$. Then

$$
\left|\frac{f(a)+f(b)}{2}-\frac{1}{b-a} \int_{a}^{b} f(x) d x\right| \leq(b-a)\left[\frac{\left|f^{\prime}(a)\right|+\left|f^{\prime}(b)\right|}{8}+\frac{\varepsilon}{4}\right] .
$$

Theorem 5 ([17, Corollary 4.9]) Let $f: I^{o} \subseteq \mathbb{R} \rightarrow \mathbb{R}$ be a differentiable mapping on $I^{o}$, $(a, b) \in I^{o} \times I^{o}, a<b$. Suppose that the function $\left|f^{\prime}\right|$ is $\alpha$-convex on $[a, b], \alpha \in(0,1]$. Then

$$
\begin{aligned}
& \left|\frac{f(a)+f(b)}{2}-\frac{1}{b-a} \int_{a}^{b} f(x) d x\right| \\
& \quad \leq \frac{(b-a)}{2(\alpha+1)(\alpha+2)}\left[\left(2^{-\alpha}+\alpha\right)\left|f^{\prime}(a)\right| \frac{\alpha(\alpha+1)+2\left(1-2^{-\alpha}\right)}{2}\left|f^{\prime}(b)\right|\right] .
\end{aligned}
$$

Theorem 6 ([17, Corollary 4.14]) Let $f: I^{o} \subseteq \mathbb{R} \rightarrow \mathbb{R}$ be a differentiable mapping on $I^{o}$, $(a, b) \in I^{o} \times I^{o}, a<b$. Suppose that the function $\left|f^{\prime}\right|$ is $h$-convex on $[a, b]$. Then

$$
\left|\frac{f(a)+f(b)}{2}-\frac{1}{b-a} \int_{a}^{b} f(x) d x\right| \leq(b-a)\left(\int_{0}^{1} h(t) d t\right)\left(\frac{\left|f^{\prime}(a)\right|+\left|f^{\prime}(b)\right|}{2}\right) .
$$

For more recent results on integral inequalities of Hermite-Hadamard type concerning the $F$-convex functions, we refer the interested reader to [19] and the references therein.

In the sequel, we recall the concepts of the left-sided and right-sided Riemann- Liouville fractional integrals of the order $\alpha>0$.

Definition 2.2 ([20]) Suppose that $f \in L([a, b])$. The left and right Riemann-Liouville fractional integrals denoted by $J_{a^{+}}^{\alpha} f$ and $J_{b-}^{\alpha} f$ of order $\alpha>0$ are defined by

$$
J_{a^{+}}^{\alpha} f(x)=\frac{1}{\Gamma(\alpha)} \int_{a}^{x}(x-t)^{\alpha-1} f(t) d t, \quad x>a,
$$

and

$$
J_{b^{-}}^{\alpha} f(x)=\frac{1}{\Gamma(\alpha)} \int_{x}^{b}(t-x)^{\alpha-1} f(t) d t, \quad x<b
$$

respectively, where $\Gamma(\alpha)$ is the gamma function defined by $\Gamma(\alpha)=\int_{0}^{\infty} e^{-t} t^{\alpha-1} d t$ and $J_{b^{-}}^{0} f(x)=J_{b^{-}}^{0} f(x)=f(x)$.

In [21], authors established the following Hermite-Hadamard type inequalities for $F$ convex functions involving a Riemann-Liouville fractional:

Theorem 7 Let $I \subseteq \mathbb{R}$ be an interval, $f: I^{o} \subseteq \mathbb{R} \rightarrow \mathbb{R}$ be a differentiable mapping on $I^{o}$, $a, b \in I^{o}, a<b$. Iff is $F$-convex on $[a, b]$, for some $F \in \mathcal{F}$, then we have

$$
\begin{aligned}
& F\left(f\left(\frac{a+b}{2}\right), \frac{\Gamma(\alpha+1)}{(b-a)^{\alpha}} J_{a^{+}}^{\alpha} f(b), \frac{\Gamma(\alpha+1)}{(b-a)^{\alpha}} J_{b^{-}}^{\alpha} f(a), \frac{1}{2}\right)+\int_{0}^{1} L_{w(t)} d t \leq 0, \\
& T_{F, w}\left(\frac{\Gamma(\alpha+1)}{(b-a)^{\alpha}}\left[J_{a^{+}}^{\alpha} f(b)+J_{b^{-}}^{\alpha} f(a)\right], f(a)+f(b), f(a)+f(b)\right)+\int_{0}^{1} L_{w(t)} d t \leq 0,
\end{aligned}
$$

where $w(t)=\alpha t^{\alpha-1}$. 
Theorem 8 Let $I \subseteq \mathbb{R}$ be an interval, $f: I^{o} \subseteq \mathbb{R} \rightarrow \mathbb{R}$ be a differentiable mapping on $I^{o}$, $a, b \in I^{o}, a<b$. If is $F$-convex on $[a, b]$, for some $F \in \mathcal{F}$ and the function $t \in(0,1) \rightarrow L_{w(t)}$ belongs to $L_{1}(a, b)$, where $w(t)=\left|(1-t)^{\alpha}-t^{\alpha}\right|$. Then we have the inequality

$$
\begin{aligned}
& T_{F, w}\left(\frac{2}{b-a}\left|\frac{f(a)+f(b)}{2}-\frac{\Gamma(\alpha+1)}{2(b-a)^{\alpha}}\left[J_{a^{+}}^{\alpha} f(b)+J_{b^{-}}^{\alpha} f(a)\right]\right|,\left|f^{\prime}(a)\right|,\left|f^{\prime}(b)\right|\right) \\
& \quad+\int_{0}^{1} L_{w(t)} d t \leq 0 .
\end{aligned}
$$

The following definitions will be useful for this study [20].

Definition 2.3 The Euler beta function is defined as follows:

$$
B(a, b)=\int_{0}^{1} t^{a-1}(1-t)^{b-1} d t, \quad a, b>0 .
$$

The incomplete beta function is defined by

$$
B_{x}(a, b)=\int_{0}^{x} t^{a-1}(1-t)^{b-1} d t, \quad a, b>0 .
$$

Note that, for $x=1$, the incomplete beta function reduces to the Euler beta function. Also, the following three lemmas are important to obtain our main results.

Lemma 1 ([22, Lemma 4]) Let $f:[a, b] \rightarrow \mathbb{R}$ be a once differentiable mappings on $(a, b)$ with $a<b, \eta(b, a)>0$. If $f^{\prime} \in L\left[a, a+e^{i \varphi} \eta(b, a)\right]$, then the following equality for the fractional integral holds:

$$
\begin{gathered}
\frac{f(a)+f\left(a+e^{i \varphi} \eta(b, a)\right)}{2}-\frac{\Gamma(\alpha+1))}{2\left(e^{i \varphi} \eta(b, a)\right)^{\alpha}}\left[J_{a^{+}}^{\alpha} f\left(a+e^{i \varphi} \eta(b, a)\right)+J_{\left(a+e^{i \varphi} \eta(b, a)\right)^{-}}^{\alpha} f(a)\right] \\
=\frac{e^{i \varphi} \eta(b, a)}{2} \int_{0}^{1}\left[(1-t)^{\alpha}-t^{\alpha}\right] f^{\prime}\left(a+(1-t) e^{i \varphi} \eta(b, a)\right) d t .
\end{gathered}
$$

Lemma 2 ([16, Lemma 5]) Let $f:[a, b] \rightarrow \mathbb{R}$ be a once differentiable mappings on $(a, b)$ with $a<b, \eta(b, a)>0$. Iff $f^{\prime \prime} \in L\left[a, a+e^{i \varphi} \eta(b, a)\right]$, then the following equality for the fractional integral holds:

$$
\begin{gathered}
\frac{f(a)+f\left(a+e^{i \varphi} \eta(b, a)\right)}{2}-\frac{\Gamma(\alpha+1))}{2\left(e^{i \varphi} \eta(b, a)\right)^{\alpha}}\left[J_{a^{+}}^{\alpha} f\left(a+e^{i \varphi} \eta(b, a)\right)+J_{\left(a+e^{i \varphi} \eta(b, a)\right)^{-}}^{\alpha} f(a)\right] \\
=\frac{\left(e^{i \varphi} \eta(b, a)\right)^{2}}{2(\alpha+1)} \int_{0}^{1}\left[1-(1-t)^{\alpha+1}-t^{\alpha+1}\right] f^{\prime \prime}\left(a+(1-t) e^{i \varphi} \eta(b, a)\right) d t .
\end{gathered}
$$

Lemma 3 ([22]) For $t \in[0,1]$, we have

$$
\begin{aligned}
& (1-t)^{m} \leq 2^{1-m}-t^{m}, \quad \text { for } m \in[0,1], \\
& (1-t)^{m} \geq 2^{1-m}-t^{m}, \quad \text { for } m \in[1, \infty) .
\end{aligned}
$$

In this study, using the $\lambda_{\varphi}$-preinvexity of the function, we establish new inequalities of Hermite-Hadamard type for differentiable function and some trapezoid type inequalities for function whose second derivatives absolutely values are $F$-convex. 


\section{Hermite-Hadamard type inequalities for differentiable functions}

In this section, we establish some inequalities of Hermite-Hadamard type for $F$-convex functions in fractional integral forms.

Theorem 9 Let $I \subseteq \mathbb{R}$ be an open invex set with respect to bifunction $\eta: I \times I \rightarrow \mathbb{R}$, where $\eta(b, a)>0$. Let $f:[0, b] \rightarrow \mathbb{R}$ be a differentiable mapping. Suppose that $\left|f^{\prime}\right|$ is measurable, decreasing, $\lambda_{\varphi}$-preinvex function on $I$, and $F$-convex on $[a, b]$, for some $F \in \mathcal{F}$ and the function $t \in(0,1) \rightarrow L_{w(t)}$ belongs to $L^{1}(0,1)$, where $w(t)=\left|(1-t)^{\alpha}-t^{\alpha}\right|$. Then

$$
\begin{aligned}
& T_{F, w}\left(\frac{2}{e^{i \varphi} \eta(b, a)} \mid \frac{f(a)+f\left(a+e^{i \varphi} \eta(b, a)\right)}{2}\right. \\
& \left.-\frac{\Gamma(\alpha+1)}{2\left(e^{i \varphi} \eta(b, a)\right)^{\alpha}}\left[J_{a^{+}}^{\alpha} f\left(a+e^{i \varphi} \eta(b, a)\right)+J_{\left(a+e^{i \varphi} \eta(b, a)\right)}^{\alpha} f(a)\right]|,| f^{\prime}(a)|,| f^{\prime}(b) \mid\right) \\
& \quad+\int_{0}^{1} L_{w(t)} d t \leq 0 .
\end{aligned}
$$

Proof Since $\left|f^{\prime}\right|$ is $F$-convex, we have

$$
F\left(\left|f^{\prime}\left(a+(1-t) e^{i \varphi} \eta(b, a)\right)\right|,\left|f^{\prime}(a)\right|,\left|f^{\prime}(b)\right|, t\right) \leq 0, \quad t \in[0,1] .
$$

Multiplying this inequality by $w(t)=\left|(1-t)^{\alpha}-t^{\alpha}\right|$ and using axiom (A3), we have

$$
F\left(w(t)\left|f^{\prime}\left(a+(1-t) e^{i \varphi} \eta(b, a)\right)\right|, w(t)\left|f^{\prime}(a)\right|, w(t)\left|f^{\prime}(b)\right|, t\right)+L_{w(t)} \leq 0, \quad t \in[0,1] .
$$

Integrating over $[0,1]$ and using axiom (A2), we get

$$
\begin{aligned}
& T_{F, w}\left(\int_{0}^{1}\left|(1-t)^{\alpha}-t^{\alpha}\right|\left|f^{\prime}\left(a+(1-t) e^{i \varphi} \eta(b, a)\right)\right| d t,\left|f^{\prime}(a)\right|,\left|f^{\prime}(b)\right|, t\right) \\
& \quad+\int_{0}^{1} L_{w(t)} d t \leq 0, \quad t \in[0,1] .
\end{aligned}
$$

But from Lemma 1 we have

$$
\begin{aligned}
& \frac{2}{e^{i \varphi} \eta(b, a)} \mid \frac{f(a)+f\left(a+e^{i \varphi} \eta(b, a)\right)}{2} \\
& \quad-\frac{\Gamma(\alpha+1))}{2\left(e^{i \varphi} \eta(b, a)\right)^{\alpha}}\left[J_{a^{+}}^{\alpha} f\left(a+e^{i \varphi} \eta(b, a)\right)+J_{\left(a+e^{i \varphi} \eta(b, a)\right)}^{\alpha} f(a)\right] \mid \\
& \leq \int_{0}^{1}\left|(1-t)^{\alpha}-t^{\alpha}\right|\left|f^{\prime}\left(a+(1-t) e^{i \varphi} \eta(b, a)\right)\right| d t .
\end{aligned}
$$

Because $T_{F, w}$ is nondecreasing with respect to the first variable so that

$$
\begin{aligned}
& T_{F, w}\left(\frac{2}{e^{i \varphi} \eta(b, a)} \mid \frac{f(a)+f\left(a+e^{i \varphi} \eta(b, a)\right)}{2}\right. \\
& \left.-\frac{\Gamma(\alpha+1)}{2\left(e^{i \varphi} \eta(b, a)\right)^{\alpha}}\left[J_{a^{+}}^{\alpha} f\left(a+e^{i \varphi} \eta(b, a)\right)+J_{\left(a+e^{i \varphi} \eta(b, a)\right)}^{\alpha} f(a)\right]|,| f^{\prime}(a)|,| f^{\prime}(b) \mid\right) \\
& \quad+\int_{0}^{1} L_{w(t)} d t \leq 0, \quad t \in[0,1] .
\end{aligned}
$$

This proves (10). 
Remark 2 If we choose $\eta(b, a)=b-a$ and $\varphi=0$ in Theorem 9, we get

$$
\begin{aligned}
& T_{F, w}\left(\frac{2}{b-a}\left|\frac{f(a)+f(b)}{2}-\frac{\Gamma(\alpha+1))}{2(b-a)^{\alpha}}\left[J_{a^{+}}^{\alpha} f(b)+J_{b^{-}}^{\alpha} f(a)\right]\right|,\left|f^{\prime}(a)\right|,\left|f^{\prime}(b)\right|\right) \\
& \quad+\int_{0}^{1} L_{w(t)} d t \leq 0 .
\end{aligned}
$$

Corollary 1 Under the assumptions of Theorem 9, if $\left|f^{\prime}\right|$ is $\varepsilon$-convex, then we have

$$
\begin{aligned}
& \left|\frac{f(a)+f\left(a+e^{i \varphi} \eta(b, a)\right)}{2}-\frac{\Gamma(\alpha+1))}{2\left(e^{i \varphi} \eta(b, a)\right)^{\alpha}}\left[J_{a^{+}}^{\alpha} f\left(a+e^{i \varphi} \eta(b, a)\right)+J_{\left(a+e^{i \varphi} \eta(b, a)\right)^{-}}^{\alpha} f(a)\right]\right| \\
& \quad \leq \frac{e^{i \varphi} \eta(b, a)}{2(\alpha+1)}\left(1-\frac{1}{2^{\alpha}}\right)\left(\left|f^{\prime}(a)\right|+\left|f^{\prime}(b)\right|+2 \varepsilon\right) .
\end{aligned}
$$

Proof Using (5) with $w(t)=\left|(1-t)^{\alpha}-t^{\alpha}\right|$, we find

$$
\begin{aligned}
\int_{0}^{1} L_{w(t)} d t & =\varepsilon \int_{0}^{1}\left(1-\left|(1-t)^{\alpha}-t^{\alpha}\right|\right) d t \\
& =\varepsilon\left[\int_{0}^{\frac{1}{2}}\left(1-(1-t)^{\alpha}+t^{\alpha}\right) d t+\int_{1}^{\frac{1}{2}}\left(1-(1-t)^{\alpha}+t^{\alpha}\right) d t\right] \\
& =\varepsilon\left[1-\frac{2}{\alpha+1}\left(1-\frac{1}{\alpha}\right)\right] .
\end{aligned}
$$

From (4) with $w(t)=\left|(1-t)^{\alpha}-t^{\alpha}\right|$, we have

$$
\begin{aligned}
& T_{F, w}\left(u_{1}, u_{2}, u_{3}\right) \\
& \quad=u_{1}-\left(\int_{0}^{1} t\left|(1-t)^{\alpha}-t^{\alpha}\right| d t\right) u_{2}-\left(\int_{0}^{1}(1-t)\left|(1-t)^{\alpha}-t^{\alpha}\right| d t\right) u_{3}-\varepsilon \\
& \quad=u_{1}-\frac{1}{\alpha+1}\left(1-\frac{1}{\alpha}\right)\left(u_{2}+u_{3}\right)-\varepsilon,
\end{aligned}
$$

for $u_{1}, u_{2}, u_{3} \in \mathbb{R}$. Hence, by Theorem 9 , we have

$$
\begin{aligned}
0 \geq & T_{F, w}\left(\frac{2}{e^{i \varphi} \eta(b, a)} \mid \frac{f(a)+f\left(a+e^{i \varphi} \eta(b, a)\right)}{2}\right. \\
& \left.-\frac{\Gamma(\alpha+1)}{2\left(e^{i \varphi} \eta(b, a)\right)^{\alpha}}\left[J_{a^{+}}^{\alpha} f\left(a+e^{i \varphi} \eta(b, a)\right)+J_{\left(a+e^{i \varphi} \eta(b, a)\right)}^{\alpha} f(a)\right]|,| f^{\prime}(a)|,| f^{\prime}(b) \mid\right) \\
& +\int_{0}^{1} L_{w(t)} d t \\
= & \frac{2}{e^{i \varphi} \eta(b, a)} \mid \frac{f(a)+f\left(a+e^{i \varphi} \eta(b, a)\right)}{2} \\
& -\frac{\Gamma(\alpha+1)}{2\left(e^{i \varphi} \eta(b, a)\right)^{\alpha}}\left[J_{a^{+}}^{\alpha} f\left(a+e^{i \varphi} \eta(b, a)\right)+J_{\left(a+e^{i \varphi} \eta(b, a)\right)}^{\alpha} f(a)\right] \mid \\
& -\frac{1}{\alpha+1}\left(1-\frac{1}{\alpha}\right)\left(\left|f^{\prime}(a)\right|+\left|f^{\prime}(b)\right|+2 \varepsilon\right) .
\end{aligned}
$$

This completes the proof. 
Remark 3 In Corollary 1 , if we choose

(a) $\eta(b, a)=b-a$ and $\varphi=0$, we get

$$
\begin{aligned}
& \left|\frac{f(a)+f(b)}{2}-\frac{\Gamma(\alpha+1))}{2(b-a)^{\alpha}}\left[J_{a^{+}}^{\alpha} f(b)+J_{b^{-}}^{\alpha} f(a)\right]\right| \\
& \quad \leq \frac{b-a}{2(\alpha+1)}\left(1-\frac{1}{2^{\alpha}}\right)\left(\left|f^{\prime}(a)\right|+\left|f^{\prime}(b)\right|+2 \varepsilon\right) .
\end{aligned}
$$

(b) $\eta(b, a)=b-a, \varphi=0$, and $\varepsilon=0$, we get

$$
\begin{gathered}
\left|\frac{f(a)+f(b)}{2}-\frac{\Gamma(\alpha+1))}{2(b-a)^{\alpha}}\left[J_{a^{+}}^{\alpha} f(b)+J_{b^{-}}^{\alpha} f(a)\right]\right| \\
\leq \frac{b-a}{2(\alpha+1)}\left(1-\frac{1}{2^{\alpha}}\right)\left(\left|f^{\prime}(a)\right|+\left|f^{\prime}(b)\right|\right)
\end{gathered}
$$

which is given by [18].

Corollary 2 Under the assumptions of Theorem 9, if $\left|f^{\prime}\right|$ is $\lambda_{\varphi}$-preinvex, then we have

$$
\begin{gathered}
\left|\frac{f(a)+f\left(a+e^{i \varphi} \eta(b, a)\right)}{2}-\frac{\Gamma(\alpha+1))}{2\left(e^{i \varphi} \eta(b, a)\right)^{\alpha}}\left[J_{a^{+}}^{\alpha} f\left(a+e^{i \varphi} \eta(b, a)\right)+J_{\left(a+e^{i \varphi} \eta(b, a)\right)^{-}}^{\alpha} f(a)\right]\right| \\
\leq \frac{e^{i \varphi} \eta(b, a)}{4}\left[B_{\frac{1}{2}}\left(\frac{1}{2}, \alpha+\frac{1}{2}\right)-B_{\frac{1}{2}}\left(\alpha+\frac{1}{2}, \frac{1}{2}\right)\right]\left(\left|f^{\prime}(a)\right|+\frac{1-\lambda}{\lambda}\left|f^{\prime}(b)\right|\right) .
\end{gathered}
$$

Proof Using (7) with $w(t)=\left|(1-t)^{\alpha}-t^{\alpha}\right|$, we have

$$
\begin{aligned}
& T_{F, w}\left(u_{1}, u_{2}, u_{3}\right) \\
& \quad=u_{1}-\left(\int_{0}^{1} \frac{\sqrt{t}}{2 \sqrt{1-t}}\left|(1-t)^{\alpha}-t^{\alpha}\right| d t\right) u_{3}-\frac{1-\lambda}{\lambda}\left(\int_{0}^{1} \frac{\sqrt{1-t}}{2 \sqrt{t}}\left|(1-t)^{\alpha}-t^{\alpha}\right| d t\right) u_{2} \\
& =u_{1}-\frac{1}{2}\left[B_{\frac{1}{2}}\left(\frac{1}{2}, \alpha+\frac{1}{2}\right)-B_{\frac{1}{2}}\left(\alpha+\frac{1}{2}, \frac{1}{2}\right)\right]\left(u_{2}+\frac{1-\lambda}{\lambda} u_{3}\right)
\end{aligned}
$$

for $u_{1}, u_{2}, u_{3} \in \mathbb{R}$. Hence, by Theorem 9 , we have

$$
\begin{aligned}
0 \geq & T_{F, w}\left(\frac{2}{e^{i \varphi} \eta(b, a)} \mid \frac{f(a)+f\left(a+e^{i \varphi} \eta(b, a)\right)}{2}\right. \\
& \left.-\frac{\Gamma(\alpha+1)}{2\left(e^{i \varphi} \eta(b, a)\right)^{\alpha}}\left[J_{a^{+}}^{\alpha} f\left(a+e^{i \varphi} \eta(b, a)\right)+J_{\left(a+e^{i \varphi} \eta(b, a)\right)}^{\alpha} f(a)\right]|,| f^{\prime}(a)|,| f^{\prime}(b) \mid\right) \\
& +\int_{0}^{1} L_{w(t)} d t \\
= & \frac{2}{e^{i \varphi} \eta(b, a)} \mid \frac{f(a)+f\left(a+e^{i \varphi} \eta(b, a)\right)}{2} \\
& -\frac{\Gamma(\alpha+1)}{2\left(e^{i \varphi} \eta(b, a)\right)^{\alpha}}\left[J_{a^{+}}^{\alpha} f\left(a+e^{i \varphi} \eta(b, a)\right)+J_{\left(a+e^{i \varphi} \eta(b, a)\right)}^{\alpha} f(a)\right] \mid \\
& -\frac{1}{2}\left[B_{\frac{1}{2}}\left(\frac{1}{2}, \alpha+\frac{1}{2}\right)-B_{\frac{1}{2}}\left(\alpha+\frac{1}{2}, \frac{1}{2}\right)\right]\left(\left|f^{\prime}(a)\right|+\frac{1-\lambda}{\lambda}\left|f^{\prime}(b)\right|\right) .
\end{aligned}
$$


This leads to

$$
\begin{aligned}
& \left|\frac{f(a)+f\left(a+e^{i \varphi} \eta(b, a)\right)}{2}-\frac{\Gamma(\alpha+1)}{2\left(e^{i \varphi} \eta(b, a)\right)^{\alpha}}\left[J_{a^{+}}^{\alpha} f\left(a+e^{i \varphi} \eta(b, a)\right)+J_{\left(a+e^{i \varphi} \eta(b, a)\right)^{-}}^{\alpha} f(a)\right]\right| \\
& \leq \frac{e^{i \varphi} \eta(b, a)}{4}\left[B_{\frac{1}{2}}\left(\frac{1}{2}, \alpha+\frac{1}{2}\right)-B_{\frac{1}{2}}\left(\alpha+\frac{1}{2}, \frac{1}{2}\right)\right]\left(\left|f^{\prime}(a)\right|+\frac{1-\lambda}{\lambda}\left|f^{\prime}(b)\right|\right) .
\end{aligned}
$$

Thus, the proof is done.

Remark 4 In Corollary 2, if we choose

(a) $\eta(b, a)=b-a$ and $\varphi=0$, we get

$$
\begin{aligned}
& \left|\frac{f(a)+f(b)}{2}-\frac{\Gamma(\alpha+1))}{2(b-a)^{\alpha}}\left[J_{a^{+}}^{\alpha} f(b)+J_{b^{-}}^{\alpha} f(a)\right]\right| \\
& \quad \leq \frac{b-a}{4}\left[B_{\frac{1}{2}}\left(\frac{1}{2}, \alpha+\frac{1}{2}\right)-B_{\frac{1}{2}}\left(\alpha+\frac{1}{2}, \frac{1}{2}\right)\right]\left(\left|f^{\prime}(a)\right|+\frac{1-\lambda}{\lambda}\left|f^{\prime}(b)\right|\right) .
\end{aligned}
$$

(b) $\eta(b, a)=b-a, \varphi=0$, and $\lambda=\frac{1}{2}$, we get

$$
\begin{aligned}
& \left|\frac{f(a)+f(b)}{2}-\frac{\Gamma(\alpha+1))}{2(b-a)^{\alpha}}\left[J_{a^{+}}^{\alpha} f(b)+J_{b^{-}}^{\alpha} f(a)\right]\right| \\
& \quad \leq \frac{b-a}{4}\left[B_{\frac{1}{2}}\left(\frac{1}{2}, \alpha+\frac{1}{2}\right)-B_{\frac{1}{2}}\left(\alpha+\frac{1}{2}, \frac{1}{2}\right)\right]\left(\left|f^{\prime}(a)\right|+\left|f^{\prime}(b)\right|\right) .
\end{aligned}
$$

Corollary 3 Under the assumptions of Theorem 9, if $\left|f^{\prime}\right|$ is h-convex, then we have

$$
\begin{aligned}
& \left|\frac{f(a)+f\left(a+e^{i \varphi} \eta(b, a)\right)}{2}-\frac{\Gamma(\alpha+1))}{2\left(e^{i \varphi} \eta(b, a)\right)^{\alpha}}\left[J_{a^{+}}^{\alpha} f\left(a+e^{i \varphi} \eta(b, a)\right)+J_{\left(a+e^{i \varphi} \eta(b, a)\right)^{-}}^{\alpha} f(a)\right]\right| \\
& \quad \leq \frac{e^{i \varphi} \eta(b, a)}{2}\left(\int_{0}^{1} h(t)\left|(1-t)^{\alpha}-t^{\alpha}\right| d t\right)\left(\left|f^{\prime}(a)\right|+\frac{1-\lambda}{\lambda}\left|f^{\prime}(b)\right|\right) .
\end{aligned}
$$

Proof Using (9) with $w(t)=\left|(1-t)^{\alpha}-t^{\alpha}\right|$, we have

$$
\begin{aligned}
& T_{F, w}\left(u_{1}, u_{2}, u_{3}\right) \\
& \quad=u_{1}-\left(\int_{0}^{1} h(t)\left|(1-t)^{\alpha}-t^{\alpha}\right| d t\right) u_{3}-\frac{1-\lambda}{\lambda}\left(\int_{0}^{1} h(1-t)\left|(1-t)^{\alpha}-t^{\alpha}\right| d t\right) u_{2} \\
& \quad=u_{1}-\left(\int_{0}^{1} h(t)\left|(1-t)^{\alpha}-t^{\alpha}\right| d t\right) u_{3}-\frac{1-\lambda}{\lambda}\left(\int_{0}^{1} h(t)\left|(1-t)^{\alpha}-t^{\alpha}\right| d t\right) u_{2} \\
& \quad=u_{1}-\left(\int_{0}^{1} h(t)\left|(1-t)^{\alpha}-t^{\alpha}\right| d t\right)\left(u_{2}+\frac{1-\lambda}{\lambda} u_{3}\right)
\end{aligned}
$$

for $u_{1}, u_{2}, u_{3} \in \mathbb{R}$. So, by Theorem 9 , we have

$$
\begin{aligned}
0 \geq & T_{F, w}\left(\frac{2}{e^{i \varphi} \eta(b, a)} \mid \frac{f(a)+f\left(a+e^{i \varphi} \eta(b, a)\right)}{2}\right. \\
& \left.-\frac{\Gamma(\alpha+1)}{2\left(e^{i \varphi} \eta(b, a)\right)^{\alpha}}\left[J_{a^{+}}^{\alpha} f\left(a+e^{i \varphi} \eta(b, a)\right)+J_{\left(a+e^{i \varphi} \eta(b, a)\right)^{-}}^{\alpha} f(a)\right]|,| f^{\prime}(a)|,| f^{\prime}(b) \mid\right)
\end{aligned}
$$




$$
\begin{aligned}
& +\int_{0}^{1} L_{w(t)} d t \\
= & \frac{2}{e^{i \varphi} \eta(b, a)} \mid \frac{f(a)+f\left(a+e^{i \varphi} \eta(b, a)\right)}{2} \\
& -\frac{\Gamma(\alpha+1)}{2\left(e^{i \varphi} \eta(b, a)\right)^{\alpha}}\left[J_{a^{+}}^{\alpha} f\left(a+e^{i \varphi} \eta(b, a)\right)+J_{\left(a+e^{i \varphi} \eta(b, a)\right)}^{\alpha} f(a)\right] \mid \\
& -\left(\int_{0}^{1} h(t)\left|(1-t)^{\alpha}-t^{\alpha}\right| d t\right)\left(\left|f^{\prime}(a)\right|+\frac{1-\lambda}{\lambda}\left|f^{\prime}(b)\right|\right) .
\end{aligned}
$$

This leads to

$$
\begin{aligned}
& \left|\frac{f(a)+f\left(a+e^{i \varphi} \eta(b, a)\right)}{2}-\frac{\Gamma(\alpha+1)}{2\left(e^{i \varphi} \eta(b, a)\right)^{\alpha}}\left[J_{a^{+}}^{\alpha} f\left(a+e^{i \varphi} \eta(b, a)\right)+J_{\left(a+e^{i \varphi} \eta(b, a)\right)}^{\alpha} f(a)\right]\right| \\
& \leq \frac{e^{i \varphi} \eta(b, a)}{2}\left(\int_{0}^{1} h(t)\left|(1-t)^{\alpha}-t^{\alpha}\right| d t\right)\left(\left|f^{\prime}(a)\right|+\frac{1-\lambda}{\lambda}\left|f^{\prime}(b)\right|\right) .
\end{aligned}
$$

Thus, the proof is done.

Theorem 10 Let $I \subseteq \mathbb{R}$ be an open invex set with respect to bifunction $\eta: I \times I \rightarrow \mathbb{R}$, where $\eta(b, a)>0$. Let $f:[0, b] \rightarrow \mathbb{R}$ be a differentiable mapping. Suppose that $\left|f^{\prime}\right|^{\frac{p}{p-1}}$ is measurable, decreasing, $\lambda_{\varphi}$-preinvex function on $I$, and $F$-convex on $[a, b]$, for some $F \in \mathcal{F}$ and $\left|f^{\prime}\right| \in L^{\frac{p}{p-1}}(a, b)$. Then

$$
T_{F, 1}\left(G_{1}(f, p),\left|f^{\prime}(a)\right|^{\frac{p}{p-1}},\left|f^{\prime}(b)\right|^{\frac{p}{p-1}}\right) \leq 0,
$$

where

$$
\begin{aligned}
G_{1}(f, p)= & \left(\frac{2}{e^{i \varphi} \eta(b, a)}\right)^{\frac{p}{p-1}}\left(\frac{\alpha p+1}{2-2^{1-\alpha p}}\right)^{\frac{1}{p-1}} \mid \frac{f(a)+f\left(a+e^{i \varphi} \eta(b, a)\right)}{2} \\
& -\left.\frac{\Gamma(\alpha+1)}{2\left(e^{i \varphi} \eta(b, a)\right)^{\alpha}}\left[J_{a^{+}}^{\alpha} f\left(a+e^{i \varphi} \eta(b, a)\right)+J_{\left(a+e^{i \varphi} \eta(b, a)\right)}^{\alpha} f(a)\right]\right|^{\frac{p}{p-1}} .
\end{aligned}
$$

Proof Since $\left|f^{\prime}\right|^{\frac{p}{p-1}}$ is $F$-convex, we have

$$
F\left(\left|f^{\prime}\left(a+(1-t) e^{i \varphi} \eta(b, a)\right)\right|^{\frac{p}{p-1}},\left|f^{\prime}(a)\right|^{\frac{p}{p-1}},\left|f^{\prime}(b)\right|^{\frac{p}{p-1}}, t\right) \leq 0, \quad t \in[0,1] .
$$

With $w(t)=1$ in (A2), we have

$$
T_{F, 1}\left(\int_{0}^{1}\left|f^{\prime}\left(a+(1-t) e^{i \varphi} \eta(b, a)\right)\right|^{\frac{p}{p-1}} d t,\left|f^{\prime}(a)\right|^{\frac{p}{p-1}},\left|f^{\prime}(b)\right|^{\frac{p}{p-1}}\right) \leq 0, \quad t \in[0,1] .
$$

Using Lemma 1 and the Hölder inequality, we get

$$
\begin{aligned}
& \left|\frac{f(a)+f\left(a+e^{i \varphi} \eta(b, a)\right)}{2}-\frac{\Gamma(\alpha+1)}{2\left(e^{i \varphi} \eta(b, a)\right)^{\alpha}}\left[J_{a^{+}}^{\alpha} f\left(a+e^{i \varphi} \eta(b, a)\right)+J_{\left(a+e^{i \varphi} \eta(b, a)\right)}^{\alpha} f(a)\right]\right| \\
& =\frac{e^{i \varphi} \eta(b, a)}{2} \int_{0}^{1}\left|(1-t)^{\alpha}-t^{\alpha}\right|\left|f^{\prime}\left(a+(1-t) e^{i \varphi} \eta(b, a)\right)\right| d t
\end{aligned}
$$




$$
\begin{aligned}
& \leq \frac{e^{i \varphi} \eta(b, a)}{2}\left(\int_{0}^{1}\left|(1-t)^{\alpha}-t^{\alpha}\right| d t\right)^{\frac{1}{p}}\left(\int_{0}^{1}\left|f^{\prime}\left(a+(1-t) e^{i \varphi} \eta(b, a)\right)\right|^{\frac{p}{p-1}} d t\right)^{\frac{p-1}{p}} \\
& =\frac{e^{i \varphi} \eta(b, a)}{2}\left(\frac{2-2^{1-\alpha p}}{\alpha p+1}\right)^{\frac{1}{p}}\left(\int_{0}^{1}\left|f^{\prime}\left(a+(1-t) e^{i \varphi} \eta(b, a)\right)\right|^{\frac{p}{p-1}} d t\right)^{\frac{p-1}{p}}
\end{aligned}
$$

or, equivalently,

$$
\begin{aligned}
& \left(\frac{2}{e^{i \varphi} \eta(b, a)}\right)^{\frac{p}{p-1}}\left(\frac{\alpha p+1}{2-2^{1-\alpha p}}\right)^{\frac{1}{p-1}} \mid \frac{f(a)+f\left(a+e^{i \varphi} \eta(b, a)\right)}{2} \\
& \quad-\left.\frac{\Gamma(\alpha+1)}{2\left(e^{i \varphi} \eta(b, a)\right)^{\alpha}}\left[J_{a^{+}}^{\alpha} f\left(a+e^{i \varphi} \eta(b, a)\right)+J_{\left(a+e^{i \varphi} \eta(b, a)\right)^{-}}^{\alpha} f(a)\right]\right|^{\frac{p}{p-1}} \\
& \leq \int_{0}^{1}\left|f^{\prime}\left(a+(1-t) e^{i \varphi} \eta(b, a)\right)\right|^{\frac{p}{p-1}} d t .
\end{aligned}
$$

Because $T_{F, 1}$ is nondecreasing with respect to the first variable, we get

$$
T_{F, 1}\left(G_{1}(f, p),\left|f^{\prime}(a)\right|^{\frac{p}{p-1}},\left|f^{\prime}(b)\right|^{\frac{p}{p-1}}\right) \leq 0
$$

Thus, the proof is completed.

Remark 5 If we choose $\eta(b, a)=b-a$ and $\varphi=0$ in Theorem 10 , we get

$$
\begin{aligned}
& T_{F, 1}\left(\left(\frac{2}{b-a}\right)^{\frac{p}{p-1}}\left(\frac{\alpha p+1}{2-2^{1-\alpha p}}\right)^{\frac{1}{p-1}}\left|\frac{f(a)+f(b)}{2}-\frac{\Gamma(\alpha+1))}{2(b-a)^{\alpha}}\left[J_{a^{+}}^{\alpha} f(b)+J_{b^{-}}^{\alpha} f(a)\right]\right|\right. \\
& \left.\left|f^{\prime}(a)\right|,\left|f^{\prime}(b)\right|\right) \leq 0 .
\end{aligned}
$$

Corollary 4 Under the assumptions of Theorem 10, if $\left|f^{\prime}\right|^{\frac{p}{p-1}}$ is $\varepsilon$-convex, we have

$$
\begin{aligned}
& \left|\frac{f(a)+f\left(a+e^{i \varphi} \eta(b, a)\right)}{2}-\frac{\Gamma(\alpha+1))}{2\left(e^{i \varphi} \eta(b, a)\right)^{\alpha}}\left[J_{a^{+}}^{\alpha} f\left(a+e^{i \varphi} \eta(b, a)\right)+J_{\left(a+e^{i \varphi} \eta(b, a)\right)^{-}}^{\alpha} f(a)\right]\right| \\
& \quad \leq \frac{e^{i \varphi} \eta(b, a)}{2}\left(\frac{2-2^{1-\alpha p}}{\alpha p+1}\right)^{\frac{1}{p}}\left(\frac{\left|f^{\prime}(a)\right|^{\frac{p}{p-1}}+\left|f^{\prime}(b)\right|^{\frac{p}{p-1}}}{2}+\varepsilon\right)^{\frac{p-1}{p}} .
\end{aligned}
$$

Proof Using (5) with $w(t)=1$, we have

$$
\int_{0}^{1} L_{w(t)} d t=\varepsilon \int_{0}^{1}(1-w(t)) d t=0
$$

From (4) with $w(t)=1$, we have

$$
\begin{aligned}
& T_{F, 1}\left(u_{1}, u_{2}, u_{3}\right) \\
& \quad=u_{1}-\left(\int_{0}^{1} t d t\right) u_{2}-\left(\int_{0}^{1}(1-t) d t\right) u_{3}-\varepsilon \\
& \quad=u_{1}-\frac{u_{2}+u_{3}}{2}-\varepsilon,
\end{aligned}
$$


for $u_{1}, u_{2}, u_{3} \in \mathbb{R}$. Hence, by Theorem 10 , we have

$$
\begin{aligned}
0 & \geq T_{F, 1}\left(G_{1}(f, p),\left|f^{\prime}(a)\right|^{\frac{p}{p-1}},\left|f^{\prime}(b)\right|^{\frac{p}{p-1}}\right) \\
& =G_{1}(f, p)-\frac{\left|f^{\prime}(a)\right|^{\frac{p}{p-1}}+\left|f^{\prime}(b)\right|^{\frac{p}{p-1}}}{2}-\varepsilon .
\end{aligned}
$$

This leads to

$$
\begin{aligned}
& \left(\frac{2}{e^{i \varphi} \eta(b, a)}\right)^{\frac{p}{p-1}}\left(\frac{\alpha p+1}{2-2^{1-\alpha p}}\right)^{\frac{1}{p-1}} \mid \frac{f(a)+f\left(a+e^{i \varphi} \eta(b, a)\right)}{2} \\
& -\left.\frac{\Gamma(\alpha+1)}{2\left(e^{i \varphi} \eta(b, a)\right)^{\alpha}}\left[J_{a^{+}}^{\alpha} f\left(a+e^{i \varphi} \eta(b, a)\right)+J_{\left(a+e^{i \varphi} \eta(b, a)\right)^{-}}^{\alpha} f(a)\right]\right|^{\frac{p}{p-1}} \\
& -\frac{\left|f^{\prime}(a)\right|^{\frac{p}{p-1}}+\left|f^{\prime}(b)\right|^{\frac{p}{p-1}}}{2} \leq 0
\end{aligned}
$$

or, equivalently,

$$
\begin{aligned}
& \left|\frac{f(a)+f\left(a+e^{i \varphi} \eta(b, a)\right)}{2}-\frac{\Gamma(\alpha+1))}{2\left(e^{i \varphi} \eta(b, a)\right)^{\alpha}}\left[J_{a^{+}}^{\alpha} f\left(a+e^{i \varphi} \eta(b, a)\right)+J_{\left(a+e^{i \varphi} \eta(b, a)\right)^{-}}^{\alpha} f(a)\right]\right| \\
& \quad \leq \frac{e^{i \varphi} \eta(b, a)}{2}\left(\frac{2-2^{1-\alpha p}}{\alpha p+1}\right)^{\frac{1}{p}}\left(\frac{\left|f^{\prime}(a)\right|^{\frac{p}{p-1}}+\left|f^{\prime}(b)\right|^{\frac{p}{p-1}}}{2}+\varepsilon\right)^{\frac{p-1}{p}} .
\end{aligned}
$$

This completes the proof.

Remark 6 In Corollary 4, if we choose

(a) $\eta(b, a)=b-a$ and $\varphi=0$, we get

$$
\begin{aligned}
& \left|\frac{f(a)+f(b)}{2}-\frac{\Gamma(\alpha+1))}{2(b-a)^{\alpha}}\left[J_{a^{+}}^{\alpha} f(b)+J_{b^{-}}^{\alpha} f(a)\right]\right| \\
& \quad \leq \frac{b-a}{2}\left(\frac{2-2^{1-\alpha p}}{\alpha p+1}\right)^{\frac{1}{p}}\left(\frac{\left|f^{\prime}(a)\right|^{\frac{p}{p-1}}+\left|f^{\prime}(b)\right|^{\frac{p}{p-1}}}{2}+\varepsilon\right)^{\frac{p-1}{p}} .
\end{aligned}
$$

(b) $\eta(b, a)=b-a, \varphi=0$, and $\varepsilon=0$, we get

$$
\begin{aligned}
& \left|\frac{f(a)+f(b)}{2}-\frac{\Gamma(\alpha+1))}{2(b-a)^{\alpha}}\left[J_{a^{+}}^{\alpha} f(b)+J_{b^{-}}^{\alpha} f(a)\right]\right| \\
& \quad \leq \frac{b-a}{2}\left(\frac{2-2^{1-\alpha p}}{\alpha p+1}\right)^{\frac{1}{p}}\left(\frac{\left|f^{\prime}(a)\right|^{\frac{p}{p-1}}+\left|f^{\prime}(b)\right|^{\frac{p}{p-1}}}{2}\right)^{\frac{p-1}{p}} .
\end{aligned}
$$

Corollary 5 Under the assumptions of Theorem 10. If $\left|f^{\prime}\right|^{\frac{p-1}{p}}$ is $\lambda_{\varphi}$-preinvex, we have

$$
\begin{aligned}
& \left|\frac{f(a)+f\left(a+e^{i \varphi} \eta(b, a)\right)}{2}-\frac{\Gamma(\alpha+1))}{2\left(e^{i \varphi} \eta(b, a)\right)^{\alpha}}\left[J_{a^{+}}^{\alpha} f\left(a+e^{i \varphi} \eta(b, a)\right)+J_{\left(a+e^{i \varphi} \eta(b, a)\right)^{-}}^{\alpha} f(a)\right]\right| \\
& \quad \leq \frac{e^{i \varphi} \eta(b, a)}{2}\left(\frac{2-2^{1-\alpha p}}{\alpha p+1}\right)^{\frac{1}{p}}\left[\frac{\pi}{4}\left(\left|f^{\prime}(a)\right|^{\frac{p-1}{p}}+\frac{1-\lambda}{\lambda}\left|f^{\prime}(b)\right|^{\frac{p-1}{p}}\right)\right]^{\frac{p-1}{p}} .
\end{aligned}
$$


Proof Using (7) with $w(t)=1$, we have

$$
\begin{aligned}
& T_{F, 1}\left(u_{1}, u_{2}, u_{3}\right) \\
& =u_{1}-\left(\int_{0}^{1} \frac{\sqrt{t}}{2 \sqrt{1-t}} d t\right) u_{3}-\frac{1-\lambda}{\lambda}\left(\int_{0}^{1} \frac{\sqrt{1-t}}{2 \sqrt{t}} d t\right) u_{2} \\
& =u_{1}-\frac{1}{2} \beta\left(\frac{1}{2}, \frac{3}{2}\right)\left(u_{2}+\frac{1-\lambda}{\lambda} u_{3}\right)=u_{1}-\frac{\pi}{4}\left(u_{2}+\frac{1-\lambda}{\lambda} u_{3}\right)
\end{aligned}
$$

for $u_{1}, u_{2}, u_{3} \in \mathbb{R}$. So, by Theorem 10 , we have

$$
\begin{aligned}
0 \geq & T_{F, 1}\left(G_{1}(f, p),\left|f^{\prime}(a)\right|^{\frac{p-1}{p}},\left|f^{\prime}(b)\right|^{\frac{p-1}{p}}\right) \\
= & \left(\frac{2}{e^{i \varphi} \eta(b, a)}\right)^{\frac{p}{p-1}}\left(\frac{\alpha p+1}{2-2^{1-\alpha p}}\right)^{\frac{1}{p-1}} \mid \frac{f(a)+f\left(a+e^{i \varphi} \eta(b, a)\right)}{2} \\
& -\left.\frac{\Gamma(\alpha+1)}{2\left(e^{i \varphi} \eta(b, a)\right)^{\alpha}}\left[J_{a^{+}}^{\alpha} f\left(a+e^{i \varphi} \eta(b, a)\right)+J_{\left(a+e^{i \varphi} \eta(b, a)\right)^{-}}^{\alpha} f(a)\right]\right|^{\frac{p}{p-1}} \\
& -\frac{\pi}{4}\left(\left|f^{\prime}(a)\right|^{\frac{p-1}{p}}+\frac{1-\lambda}{\lambda}\left|f^{\prime}(b)\right|^{\frac{p-1}{p}}\right) .
\end{aligned}
$$

This leads to

$$
\begin{aligned}
& \left|\frac{f(a)+f\left(a+e^{i \varphi} \eta(b, a)\right)}{2}-\frac{\Gamma(\alpha+1)}{2\left(e^{i \varphi} \eta(b, a)\right)^{\alpha}}\left[J_{a^{+}}^{\alpha} f\left(a+e^{i \varphi} \eta(b, a)\right)+J_{\left(a+e^{i \varphi} \eta(b, a)\right)^{-}}^{\alpha} f(a)\right]\right| \\
& \leq \frac{e^{i \varphi} \eta(b, a)}{2}\left(\frac{2-2^{1-\alpha p}}{\alpha p+1}\right)^{\frac{1}{p}}\left[\frac{\pi}{4}\left(\left|f^{\prime}(a)\right|^{\frac{p-1}{p}}+\frac{1-\lambda}{\lambda}\left|f^{\prime}(b)\right|^{\frac{p-1}{p}}\right)\right]^{\frac{p-1}{p}} .
\end{aligned}
$$

Thus, the proof is done.

Remark 7 In Corollary 5, if we choose

(a) $\eta(b, a)=b-a$ and $\varphi=0$, we get

$$
\begin{aligned}
& \left|\frac{f(a)+f(b)}{2}-\frac{\Gamma(\alpha+1))}{2(b-a)^{\alpha}}\left[J_{a^{+}}^{\alpha} f(b)+J_{b^{-}}^{\alpha} f(a)\right]\right| \\
& \quad \leq \frac{b-a}{2}\left(\frac{2-2^{1-\alpha p}}{\alpha p+1}\right)^{\frac{1}{p}}\left[\frac{\pi}{4}\left(\left|f^{\prime}(a)\right|^{\frac{p-1}{p}}+\frac{1-\lambda}{\lambda}\left|f^{\prime}(b)\right|^{\frac{p-1}{p}}\right)\right]^{\frac{p-1}{p}} .
\end{aligned}
$$

(b) $\eta(b, a)=b-a, \varphi=0$, and $\lambda=\frac{1}{2}$, we get

$$
\begin{aligned}
& \left|\frac{f(a)+f(b)}{2}-\frac{\Gamma(\alpha+1))}{2(b-a)^{\alpha}}\left[J_{a^{+}}^{\alpha} f(b)+J_{b^{-}}^{\alpha} f(a)\right]\right| \\
& \quad \leq \frac{b-a}{2}\left(\frac{2-2^{1-\alpha p}}{\alpha p+1}\right)^{\frac{1}{p}}\left[\frac{\pi}{4}\left(\left|f^{\prime}(a)\right|^{\frac{p-1}{p}}+\left|f^{\prime}(b)\right|^{\frac{p-1}{p}}\right)\right]^{\frac{p-1}{p}} .
\end{aligned}
$$


Corollary 6 Under the assumptions of Theorem 10. If $\left|f^{\prime}\right|^{\frac{p}{p-1}}$ is h-convex, we have

$$
\begin{aligned}
& \left|\frac{f(a)+f\left(a+e^{i \varphi} \eta(b, a)\right)}{2}-\frac{\Gamma(\alpha+1))}{2\left(e^{i \varphi} \eta(b, a)\right)^{\alpha}}\left[J_{a^{+}}^{\alpha} f\left(a+e^{i \varphi} \eta(b, a)\right)+J_{\left(a+e^{i \varphi} \eta(b, a)\right)^{-}}^{\alpha} f(a)\right]\right| \\
& \leq \frac{e^{i \varphi} \eta(b, a)}{2}\left(\frac{2-2^{1-\alpha p}}{\alpha p+1}\right)^{\frac{1}{p}}\left(\int_{0}^{1} h(t)\right)^{\frac{p-1}{p}}\left(\left|f^{\prime}(a)\right|^{\frac{p-1}{p}}+\frac{1-\lambda}{\lambda}\left|f^{\prime}(b)\right|^{\frac{p-1}{p}}\right) .
\end{aligned}
$$

Proof From (9) with $w(t)=1$, we have

$$
\begin{aligned}
& T_{F, 1}\left(u_{1}, u_{2}, u_{3}\right) \\
& =u_{1}-\left(\int_{0}^{1} h(t) d t\right) u_{3}-\frac{1-\lambda}{\lambda}\left(\int_{0}^{1} h(1-t) d t\right) u_{2} \\
& =u_{1}-\left(\int_{0}^{1} h(t) d t\right) u_{3}-\frac{1-\lambda}{\lambda}\left(\int_{0}^{1} h(t) d t\right) u_{2} \\
& =u_{1}-\left(\int_{0}^{1} h(t) d t\right)\left(u_{2}+\frac{1-\lambda}{\lambda} u_{3}\right)
\end{aligned}
$$

for $u_{1}, u_{2}, u_{3} \in \mathbb{R}$. So, by Theorem 9 , we have

$$
\begin{aligned}
0 \geq & T_{F, 1}\left(G_{1}(f, p),\left|f^{\prime}(a)\right|^{\frac{p-1}{p}},\left|f^{\prime}(b)\right|^{\frac{p-1}{p}}\right) \\
= & \left(\frac{2}{e^{i \varphi} \eta(b, a)}\right)^{\frac{p}{p-1}}\left(\frac{\alpha p+1}{2-2^{1-\alpha p}}\right)^{\frac{1}{p-1}} \\
& \times\left|\frac{f(a)+f\left(a+e^{i \varphi} \eta(b, a)\right)}{2}-\frac{\Gamma(\alpha+1)}{2\left(e^{i \varphi} \eta(b, a)\right)^{\alpha}}\left[J_{a^{+}}^{\alpha} f\left(a+e^{i \varphi} \eta(b, a)\right)+J_{\left(a+e^{i \varphi} \eta(b, a)\right)^{-}}^{\alpha} f(a)\right]\right| \\
& -\left(\int_{0}^{1} h(t) d t\right)\left(\left|f^{\prime}(a)\right|^{\frac{p-1}{p}}+\frac{1-\lambda}{\lambda}\left|f^{\prime}(b)\right|^{\frac{p-1}{p}}\right),
\end{aligned}
$$

that is,

$$
\begin{aligned}
& \left|\frac{f(a)+f\left(a+e^{i \varphi} \eta(b, a)\right)}{2}-\frac{\Gamma(\alpha+1)}{2\left(e^{i \varphi} \eta(b, a)\right)^{\alpha}}\left[J_{a^{+}}^{\alpha} f\left(a+e^{i \varphi} \eta(b, a)\right)+J_{\left(a+e^{i \varphi} \eta(b, a)\right)^{-}}^{\alpha} f(a)\right]\right| \\
& \leq \frac{e^{i \varphi} \eta(b, a)}{2}\left(\frac{2-2^{1-\alpha p}}{\alpha p+1}\right)^{\frac{1}{p}} \\
& \quad \times\left(\int_{0}^{1} h(t)\left|(1-t)^{\alpha}-t^{\alpha}\right| d t\right)\left(\left|f^{\prime}(a)\right|^{\frac{p-1}{p}}+\frac{1-\lambda}{\lambda}\left|f^{\prime}(b)\right|^{\frac{p-1}{p}}\right) .
\end{aligned}
$$

This completes the proof.

Theorem 11 Let $I \subseteq \mathbb{R}$ be an open invex set with respect to bifunction $\eta: I \times I \rightarrow \mathbb{R}$, where $\eta(b, a)>0$. Let $f:[0, b] \rightarrow \mathbb{R}$ be a differentiable mapping. Suppose that $\left.\left.\right|^{\prime}\right|^{\frac{p}{p-1}}$ is measurable, decreasing, $\lambda_{\varphi}$-preinvex function on $I$, and $F$-convex on $[a, b]$, for some $F \in \mathcal{F}$ and $\left|f^{\prime}\right| \in L^{\frac{p}{p-1}}(a, b)$. Then

$$
T_{F, w}\left(G_{2}(f, p),\left|f^{\prime}(a)\right|^{\frac{p}{p-1}},\left|f^{\prime}(b)\right|^{\frac{p}{p-1}}\right)+\int_{0}^{1} L_{w(t)} d t \leq 0
$$


where

$$
\begin{aligned}
G_{2}(f, p)= & \left(\frac{2}{e^{i \varphi} \eta(b, a)}\right)^{\frac{p}{p-1}}\left(\frac{\alpha+1}{2-2^{1-\alpha}}\right)^{\frac{1}{p-1}} \mid \frac{f(a)+f\left(a+e^{i \varphi} \eta(b, a)\right)}{2} \\
& -\left.\frac{\Gamma(\alpha+1)}{2\left(e^{i \varphi} \eta(b, a)\right)^{\alpha}}\left[J_{a^{\alpha}}^{\alpha} f\left(a+e^{i \varphi} \eta(b, a)\right)+J_{\left(a+e^{i \varphi} \eta(b, a)\right)}^{\alpha} f(a)\right]\right|^{\frac{p}{p-1}}
\end{aligned}
$$

for $w(t)=\left|(1-t)^{\alpha}-t^{\alpha}\right|$.

Proof Since $\left|f^{\prime}\right|^{\frac{p}{p-1}}$ is $F$-convex, we have

$$
F\left(\left|f^{\prime}\left(a+(1-t) e^{i \varphi} \eta(b, a)\right)\right|^{\frac{p}{p-1}},\left|f^{\prime}(a)\right|^{\frac{p}{p-1}},\left|f^{\prime}(b)\right|^{\frac{p}{p-1}}, t\right) \leq 0, \quad t \in[0,1] .
$$

Using (A3) with $w(t)=\left|(1-t)^{\alpha}-t^{\alpha}\right|$, we obtain

$$
\begin{aligned}
& F\left(w(t)\left|f^{\prime}\left(a+(1-t) e^{i \varphi} \eta(b, a)\right)\right|^{\frac{p}{p-1}}, w(t)\left|f^{\prime}(a)\right|^{\frac{p}{p-1}}, w(t)\left|f^{\prime}(b)\right|^{\frac{p}{p-1}}, t\right)+L_{w(t)} \leq 0, \\
& \quad t \in[0,1] .
\end{aligned}
$$

Integrating over $[0,1]$ and using axiom (A2), we obtain

$$
\begin{aligned}
& T_{F, w}\left(\int_{0}^{1} w(t)\left|f^{\prime}\left(a+(1-t) e^{i \varphi} \eta(b, a)\right)\right|^{\frac{p}{p-1}} d t,\left|f^{\prime}(a)\right|^{\frac{p}{p-1}},\left|f^{\prime}(b)\right|^{\frac{p}{p-1}}\right)+\int_{0}^{1} L_{w(t)} d t \leq 0, \\
& \quad t \in[0,1] .
\end{aligned}
$$

Using Lemma 1 and the power mean inequality, we get

$$
\begin{aligned}
& \left|\frac{f(a)+f\left(a+e^{i \varphi} \eta(b, a)\right)}{2}-\frac{\Gamma(\alpha+1)}{2\left(e^{i \varphi} \eta(b, a)\right)^{\alpha}}\left[J_{a^{+}}^{\alpha} f\left(a+e^{i \varphi} \eta(b, a)\right)+J_{\left(a+e^{i \varphi} \eta(b, a)\right)}^{\alpha} f(a)\right]\right| \\
& \quad=\frac{e^{i \varphi} \eta(b, a)}{2} \int_{0}^{1}\left|(1-t)^{\alpha}-t^{\alpha}\right|\left|f^{\prime}\left(a+(1-t) e^{i \varphi} \eta(b, a)\right)\right| d t \\
& \quad \leq \frac{e^{i \varphi} \eta(b, a)}{2}\left(\int_{0}^{1}\left|(1-t)^{\alpha}-t^{\alpha}\right| d t\right)^{\frac{1}{p}}\left(\int_{0}^{1} w(t)\left|f^{\prime}\left(a+(1-t) e^{i \varphi} \eta(b, a)\right)\right|^{\frac{p}{p-1}} d t\right)^{\frac{p-1}{p}} \\
& \quad=\frac{e^{i \varphi} \eta(b, a)}{2}\left(\frac{2-2^{1-\alpha}}{\alpha+1}\right)^{\frac{1}{p}}\left(\int_{0}^{1} w(t)\left|f^{\prime}\left(a+(1-t) e^{i \varphi} \eta(b, a)\right)\right|^{\frac{p}{p-1}} d t\right)^{\frac{p-1}{p}}
\end{aligned}
$$

or, equivalently,

$$
\begin{aligned}
& \left(\frac{2}{e^{i \varphi} \eta(b, a)}\right)^{\frac{p}{p-1}}\left(\frac{\alpha+1}{2-2^{1-\alpha}}\right)^{\frac{1}{p-1}} \mid \frac{f(a)+f\left(a+e^{i \varphi} \eta(b, a)\right)}{2} \\
& \quad-\left.\frac{\Gamma(\alpha+1)}{2\left(e^{i \varphi} \eta(b, a)\right)^{\alpha}}\left[J_{a^{+}}^{\alpha} f\left(a+e^{i \varphi} \eta(b, a)\right)+J_{\left(a+e^{i \varphi} \eta(b, a)\right)}^{\alpha} f(a)\right]\right|^{\frac{p}{p-1}} \\
& \quad \leq \int_{0}^{1} w(t)\left|f^{\prime}\left(a+(1-t) e^{i \varphi} \eta(b, a)\right)\right|^{\frac{p}{p-1}} d t .
\end{aligned}
$$


Because $T_{F, w}$ is nondecreasing with respect to the first variable, we find

$$
T_{F, w}\left(G_{2}(f, p),\left|f^{\prime}(a)\right|^{\frac{p}{p-1}},\left|f^{\prime}(b)\right|^{\frac{p}{p-1}}\right)+\int_{0}^{1} L_{w(t)} d t \leq 0 .
$$

This completes the proof.

Remark 8 If we choose $\eta(b, a)=b-a$ and $\varphi=0$ in Theorem 11 , we get

$$
\begin{aligned}
& T_{F, w}\left(\left(\frac{2}{b-a}\right)^{\frac{p}{p-1}}\left(\frac{\alpha+1}{2-2^{1-\alpha}}\right)^{\frac{1}{p-1}}\left|\frac{f(a)+f(b)}{2}-\frac{\Gamma(\alpha+1))}{2(b-a)^{\alpha}}\left[J_{a^{+}}^{\alpha} f(b)+J_{b^{-}}^{\alpha} f(a)\right]\right|\right. \\
& \left.\left|f^{\prime}(a)\right|,\left|f^{\prime}(b)\right|\right)+\int_{0}^{1} L_{w(t)} d t \leq 0 .
\end{aligned}
$$

Corollary 7 Under the assumptions of Theorem 11, if $\left|f^{\prime}\right|^{\frac{p}{p-1}}$ is $\varepsilon$-convex, we have

$$
\begin{aligned}
& \left|\frac{f(a)+f\left(a+e^{i \varphi} \eta(b, a)\right)}{2}-\frac{\Gamma(\alpha+1))}{2\left(e^{i \varphi} \eta(b, a)\right)^{\alpha}}\left[J_{a^{+}}^{\alpha} f\left(a+e^{i \varphi} \eta(b, a)\right)+J_{\left(a+e^{i \varphi} \eta(b, a)\right)^{-}}^{\alpha} f(a)\right]\right| \\
& \leq \frac{e^{i \varphi} \eta(b, a)}{2}\left(\frac{2-2^{1-\alpha}}{\alpha+1}\right)^{\frac{1}{p}}\left[\frac{2^{\alpha}-1}{2^{\alpha}(\alpha+1)}\left(\left|f^{\prime}(a)\right|^{\frac{p}{p-1}}+\left|f^{\prime}(b)\right|^{\frac{p}{p-1}}\right)+2 \varepsilon\right]^{\frac{p-1}{p}} .
\end{aligned}
$$

Proof Using (5) with $w(t)=\left|(1-t)^{\alpha}-t^{\alpha}\right|$, we get

$$
\int_{0}^{1} L_{w(t)} d t=\varepsilon\left(1-2 \frac{2^{\alpha}-1}{2^{\alpha}(\alpha+1)}\right) .
$$

From (4) with $w(t)=\left|(1-t)^{\alpha}-t^{\alpha}\right|$, we get

$$
\begin{aligned}
& T_{F, w}\left(u_{1}, u_{2}, u_{3}\right) \\
& \quad=u_{1}-\left(\int_{0}^{1}\left|(1-t)^{\alpha}-t^{\alpha}\right| t d t\right) u_{2}-\left(\int_{0}^{1}\left|(1-t)^{\alpha}-t^{\alpha}\right|(1-t) d t\right) u_{3}-\varepsilon \\
& \quad=u_{1}-\frac{2^{\alpha}-1}{2^{\alpha}(\alpha+1)}\left(u_{2}+u_{3}\right)-\varepsilon,
\end{aligned}
$$

for $u_{1}, u_{2}, u_{3} \in \mathbb{R}$. Hence, by Theorem 10 , we have

$$
\begin{aligned}
0 & \geq T_{F, w}\left(G_{2}(f, p),\left|f^{\prime}(a)\right|^{\frac{p}{p-1}},\left|f^{\prime}(b)\right|^{\frac{p}{p-1}}\right)+\int_{0}^{1} L_{w(t)} d t \\
& =G_{2}(f, p)-\frac{2^{\alpha}-1}{2^{\alpha}(\alpha+1)}\left(\left|f^{\prime}(a)\right|^{\frac{p}{p-1}}+\left|f^{\prime}(b)\right|^{\frac{p}{p-1}}\right)-\varepsilon+\varepsilon\left(1-2 \frac{2^{\alpha}-1}{2^{\alpha}(\alpha+1)}\right) .
\end{aligned}
$$

This implies that

$$
\begin{aligned}
& \left|\frac{f(a)+f\left(a+e^{i \varphi} \eta(b, a)\right)}{2}-\frac{\Gamma(\alpha+1))}{2\left(e^{i \varphi} \eta(b, a)\right)^{\alpha}}\left[J_{a^{+}}^{\alpha} f\left(a+e^{i \varphi} \eta(b, a)\right)+J_{\left(a+e^{i \varphi} \eta(b, a)\right)^{-}}^{\alpha} f(a)\right]\right| \\
& \leq \frac{e^{i \varphi} \eta(b, a)}{2}\left(\frac{2-2^{1-\alpha}}{\alpha+1}\right)^{\frac{1}{p}}\left[\frac{2^{\alpha}-1}{2^{\alpha}(\alpha+1)}\left(\left|f^{\prime}(a)\right|^{\frac{p}{p-1}}+\left|f^{\prime}(b)\right|^{\frac{p}{p-1}}\right)+2 \varepsilon\right]^{\frac{p-1}{p}} .
\end{aligned}
$$

This completes the proof. 
Remark 9 In Corollary 7, if we choose

(a) $\eta(b, a)=b-a$ and $\varphi=0$, we get

$$
\begin{aligned}
& \left|\frac{f(a)+f(b)}{2}-\frac{\Gamma(\alpha+1))}{2(b-a)^{\alpha}}\left[J_{a^{+}}^{\alpha} f(b)+J_{b^{-}}^{\alpha} f(a)\right]\right| \\
& \quad \leq \frac{b-a}{2}\left(\frac{2-2^{1-\alpha}}{\alpha+1}\right)^{\frac{1}{p}}\left[\frac{2^{\alpha}-1}{2^{\alpha}(\alpha+1)}\left(\left|f^{\prime}(a)\right|^{\frac{p}{p-1}}+\left|f^{\prime}(b)\right|^{\frac{p}{p-1}}\right)+2 \varepsilon\right]^{\frac{p-1}{p}} .
\end{aligned}
$$

(b) $\eta(b, a)=b-a, \varphi=0$, and $\varepsilon=0$, we get

$$
\begin{aligned}
& \left|\frac{f(a)+f(b)}{2}-\frac{\Gamma(\alpha+1))}{2(b-a)^{\alpha}}\left[J_{a^{+}}^{\alpha} f(b)+J_{b^{-}}^{\alpha} f(a)\right]\right| \\
& \quad \leq \frac{b-a}{2}\left(\frac{2-2^{1-\alpha}}{\alpha+1}\right)^{\frac{1}{p}}\left[\frac{2^{\alpha}-1}{2^{\alpha}(\alpha+1)}\left(\left|f^{\prime}(a)\right|^{\frac{p}{p-1}}+\left|f^{\prime}(b)\right|^{\frac{p}{p-1}}\right)\right]^{\frac{p-1}{p}} .
\end{aligned}
$$

Corollary 8 Under the assumptions of Theorem 11. If $\left|f^{\prime}\right|^{\frac{p-1}{p}}$ is $\lambda_{\varphi}$-preinvex, we have

$$
\begin{aligned}
& \mid \frac{f(a)+f\left(a+e^{i \varphi} \eta(b, a)\right)}{2}-\frac{\Gamma(\alpha+1))}{2\left(e^{i \varphi} \eta(b, a)\right)^{\alpha}}\left[J_{a^{+}}^{\alpha} f\left(a+e^{i \varphi} \eta(b, a)\right)+J_{\left(a+e^{i \varphi} \eta(b, a)\right)^{-}}^{\alpha} f(a)\right] \mid \\
& \leq \frac{e^{i \varphi} \eta(b, a)}{2}\left(\frac{2-2^{1-\alpha}}{\alpha+1}\right)^{\frac{1}{p}} \\
& \quad \times\left[\frac{B_{\frac{1}{2}}\left(\frac{1}{2}, \alpha+\frac{1}{2}\right)-B_{\frac{1}{2}}\left(\alpha+\frac{1}{2}, \frac{1}{2}\right)}{2}\left(\left|f^{\prime}(a)\right|^{\frac{p}{p-1}}+\frac{1-\lambda}{\lambda}\left|f^{\prime}(b)\right|^{\frac{p}{p-1}}\right)\right]^{\frac{p-1}{p}} .
\end{aligned}
$$

Proof Using (7) with $w(t)=\left|(1-t)^{\alpha}-t^{\alpha}\right|$, we have

$$
\begin{aligned}
& T_{F, w}\left(u_{1}, u_{2}, u_{3}\right) \\
& \quad=u_{1}-\left(\int_{0}^{1} \frac{\sqrt{t}}{2 \sqrt{1-t}}\left|(1-t)^{\alpha}-t^{\alpha}\right| d t\right) u_{3}-\frac{1-\lambda}{\lambda}\left(\int_{0}^{1} \frac{\sqrt{1-t}}{2 \sqrt{t}}\left|(1-t)^{\alpha}-t^{\alpha}\right| d t\right) u_{2} \\
& \quad=u_{1}-\frac{1}{2}\left(B_{\frac{1}{2}}\left(\frac{1}{2}, \alpha+\frac{1}{2}\right)-B_{\frac{1}{2}}\left(\alpha+\frac{1}{2}, \frac{1}{2}\right)\right)\left(u_{2}+\frac{1-\lambda}{\lambda} u_{3}\right)
\end{aligned}
$$

for $u_{1}, u_{2}, u_{3} \in \mathbb{R}$. Now, by Theorem 11 , we have

$$
\begin{aligned}
0 \geq & T_{F, w}\left(G_{2}(f, p),\left|f^{\prime}(a)\right|^{\frac{p-1}{p}},\left|f^{\prime}(b)\right|^{\frac{p-1}{p}}\right) \\
= & \left(\frac{2}{e^{i \varphi} \eta(b, a)}\right)^{\frac{p}{p-1}}\left(\frac{\alpha+1}{2-2^{1-\alpha}}\right)^{\frac{1}{p-1}} \\
& \times\left|\frac{f(a)+f\left(a+e^{i \varphi} \eta(b, a)\right)}{2}-\frac{\Gamma(\alpha+1)}{2\left(e^{i \varphi} \eta(b, a)\right)^{\alpha}}\left[J_{a^{+}}^{\alpha} f\left(a+e^{i \varphi} \eta(b, a)\right)+J_{\left(a+e^{i \varphi} \eta(b, a)\right)}^{\alpha} f(a)\right]\right| \\
& -\frac{1}{2}\left(B_{\frac{1}{2}}\left(\frac{1}{2}, \alpha+\frac{1}{2}\right)-B_{\frac{1}{2}}\left(\alpha+\frac{1}{2}, \frac{1}{2}\right)\right)\left(\left|f^{\prime}(a)\right|^{\frac{p-1}{p}}+\frac{1-\lambda}{\lambda}\left|f^{\prime}(b)\right|^{\frac{p-1}{p}}\right) .
\end{aligned}
$$

This leads to

$$
\left|\frac{f(a)+f\left(a+e^{i \varphi} \eta(b, a)\right)}{2}-\frac{\Gamma(\alpha+1)}{2\left(e^{i \varphi} \eta(b, a)\right)^{\alpha}}\left[J_{a^{+}}^{\alpha} f\left(a+e^{i \varphi} \eta(b, a)\right)+J_{\left(a+e^{i \varphi} \eta(b, a)\right)}^{\alpha} f(a)\right]\right|
$$




$$
\begin{aligned}
\leq & \frac{e^{i \varphi} \eta(b, a)}{2}\left(\frac{2-2^{1-\alpha}}{\alpha+1}\right)^{\frac{1}{p}} \\
& \times\left[\frac{B_{\frac{1}{2}}\left(\frac{1}{2}, \alpha+\frac{1}{2}\right)-B_{\frac{1}{2}}\left(\alpha+\frac{1}{2}, \frac{1}{2}\right)}{2}\left(\left|f^{\prime}(a)\right|^{\frac{p}{p-1}}+\frac{1-\lambda}{\lambda}\left|f^{\prime}(b)\right|^{\frac{p}{p-1}}\right)\right]^{\frac{p-1}{p}} .
\end{aligned}
$$

Thus, the proof is done.

Remark 10 In Corollary 8, if we choose

(a) $\eta(b, a)=b-a$ and $\varphi=0$, we get

$$
\begin{aligned}
& \left|\frac{f(a)+f(b)}{2}-\frac{\Gamma(\alpha+1))}{2(b-a)^{\alpha}}\left[J_{a^{f}}^{\alpha} f(b)+J_{b^{-}}^{\alpha} f(a)\right]\right| \\
& \leq \frac{b-a}{2}\left(\frac{2-2^{1-\alpha}}{\alpha+1}\right)^{\frac{1}{p}} \\
& \quad \times\left[\frac{B_{\frac{1}{2}}\left(\frac{1}{2}, \alpha+\frac{1}{2}\right)-B_{\frac{1}{2}}\left(\alpha+\frac{1}{2}, \frac{1}{2}\right)}{2}\left(\left|f^{\prime}(a)\right|^{\frac{p}{p-1}}+\frac{1-\lambda}{\lambda}\left|f^{\prime}(b)\right|^{\frac{p}{p-1}}\right)\right]^{\frac{p-1}{p}} .
\end{aligned}
$$

(b) $\eta(b, a)=b-a, \varphi=0$, and $\lambda=\frac{1}{2}$, we get

$$
\begin{aligned}
& \left|\frac{f(a)+f(b)}{2}-\frac{\Gamma(\alpha+1))}{2(b-a)^{\alpha}}\left[J_{a^{+}}^{\alpha} f(b)+J_{b^{-}}^{\alpha} f(a)\right]\right| \\
& \leq \frac{b-a}{2}\left(\frac{2-2^{1-\alpha}}{\alpha+1}\right)^{\frac{1}{p}} \\
& \quad \times\left[\frac{B_{\frac{1}{2}}\left(\frac{1}{2}, \alpha+\frac{1}{2}\right)-B_{\frac{1}{2}}\left(\alpha+\frac{1}{2}, \frac{1}{2}\right)}{2}\left(\left|f^{\prime}(a)\right|^{\frac{p}{p-1}}+\left|f^{\prime}(b)\right|^{\frac{p}{p-1}}\right)\right]^{\frac{p-1}{p}} .
\end{aligned}
$$

Corollary 9 Under the assumptions of Theorem 11. If $\left.\left.\right|^{\prime}\right|^{\frac{p}{p-1}}$ is $h$-convex, we have

$$
\begin{aligned}
& \left|\frac{f(a)+f\left(a+e^{i \varphi} \eta(b, a)\right)}{2}-\frac{\Gamma(\alpha+1))}{2\left(e^{i \varphi} \eta(b, a)\right)^{\alpha}}\left[J_{a^{+}}^{\alpha} f\left(a+e^{i \varphi} \eta(b, a)\right)+J_{\left(a+e^{i \varphi} \eta(b, a)\right)^{-}}^{\alpha} f(a)\right]\right| \\
& \leq \frac{e^{i \varphi} \eta(b, a)}{2}\left(\frac{2-2^{1-\alpha}}{\alpha+1}\right)^{\frac{1}{p}} \\
& \quad \times\left(\int_{0}^{1} h(t)\left|(1-t)^{\alpha}-t^{\alpha}\right| d t\right)^{\frac{p-1}{p}}\left(\left|f^{\prime}(a)\right|^{\frac{p}{p-1}}+\frac{1-\lambda}{\lambda}\left|f^{\prime}(b)\right|^{\frac{p}{p-1}}\right)^{\frac{p-1}{p}} .
\end{aligned}
$$

Proof From (9) with $w(t)=\left|(1-t)^{\alpha}-t^{\alpha}\right|$, we have

$$
\begin{aligned}
& T_{F, w}\left(u_{1}, u_{2}, u_{3}\right) \\
& \quad=u_{1}-\left(\int_{0}^{1} h(t)\left|(1-t)^{\alpha}-t^{\alpha}\right| d t\right) u_{3}-\frac{1-\lambda}{\lambda}\left(\int_{0}^{1} h(1-t)\left|(1-t)^{\alpha}-t^{\alpha}\right| d t\right) u_{2} \\
& \quad=u_{1}-\left(\int_{0}^{1} h(t)\left|(1-t)^{\alpha}-t^{\alpha}\right| d t\right) u_{3}-\frac{1-\lambda}{\lambda}\left(\int_{0}^{1} h(t)\left|(1-t)^{\alpha}-t^{\alpha}\right| d t\right) u_{2} \\
& \quad=u_{1}-\left(\int_{0}^{1} h(t)\left|(1-t)^{\alpha}-t^{\alpha}\right| d t\right)\left(u_{2}+\frac{1-\lambda}{\lambda} u_{3}\right)
\end{aligned}
$$


for $u_{1}, u_{2}, u_{3} \in \mathbb{R}$. So, by Theorem 11 , we have

$$
\begin{aligned}
0 \geq & T_{F, w}\left(G_{2}(f, p),\left|f^{\prime}(a)\right|^{\frac{p-1}{p}},\left|f^{\prime}(b)\right|^{\frac{p-1}{p}}\right) \\
= & \left(\frac{2}{e^{i \varphi} \eta(b, a)}\right)^{\frac{p}{p-1}}\left(\frac{\alpha+1}{2-2^{1-\alpha}}\right)^{\frac{1}{p-1}} \\
& \times\left|\frac{f(a)+f\left(a+e^{i \varphi} \eta(b, a)\right)}{2}-\frac{\Gamma(\alpha+1)}{2\left(e^{i \varphi} \eta(b, a)\right)^{\alpha}}\left[J_{a^{+}}^{\alpha} f\left(a+e^{i \varphi} \eta(b, a)\right)+J_{\left(a+e^{i \varphi} \eta(b, a)\right)^{-}}^{\alpha} f(a)\right]\right| \\
& -\left(\int_{0}^{1} h(t) d t\right)\left(\left|f^{\prime}(a)\right|^{\frac{p-1}{p}}+\frac{1-\lambda}{\lambda}\left|f^{\prime}(b)\right|^{\frac{p-1}{p}}\right),
\end{aligned}
$$

that is,

$$
\begin{aligned}
& \left|\frac{f(a)+f\left(a+e^{i \varphi} \eta(b, a)\right)}{2}-\frac{\Gamma(\alpha+1)}{2\left(e^{i \varphi} \eta(b, a)\right)^{\alpha}}\left[J_{a^{+}}^{\alpha} f\left(a+e^{i \varphi} \eta(b, a)\right)+J_{\left(a+e^{i \varphi} \eta(b, a)\right)^{-}}^{\alpha} f(a)\right]\right| \\
& \leq \frac{e^{i \varphi} \eta(b, a)}{2}\left(\frac{2-2^{1-\alpha}}{\alpha+1}\right)^{\frac{1}{p}} \\
& \quad \times\left(\int_{0}^{1} h(t)\left|(1-t)^{\alpha}-t^{\alpha}\right| d t\right)^{\frac{p-1}{p}}\left(\left|f^{\prime}(a)\right|^{\frac{p}{p-1}}+\frac{1-\lambda}{\lambda}\left|f^{\prime}(b)\right|^{\frac{p}{p-1}}\right)^{\frac{p-1}{p}} .
\end{aligned}
$$

This completes the proof.

\section{Trapezoid type inequalities for twice differentiable functions}

In this section, we establish some trapezoid type inequalities for functions whose second derivatives absolutely values are

Theorem 12 Let $f:[0, b] \rightarrow \mathbb{R}$ be a differentiable mapping and $\left|f^{\prime \prime}\right|$ is measurable, decreasing, $\lambda_{\varphi}$-preinvex function on $[0, b]$ for $0 \leq a<b, \eta(b, a)>0$ and $\alpha>0$. Suppose that $F$-convex on $[0, b]$, for some $F \in \mathcal{F}$ and the function $t \in(0,1) \rightarrow L_{w(t)}$ belongs to $L^{1}(0,1)$, where $w(t)=1-(1-t)^{\alpha+1}-t^{\alpha+1}$. Then

$$
\begin{aligned}
& T_{F, w}\left(\frac{2(\alpha+1)}{\left(e^{i \varphi} \eta(b, a)\right)^{2}} \mid \frac{f(a)+f\left(a+e^{i \varphi} \eta(b, a)\right)}{2}\right. \\
& \left.\quad-\frac{\Gamma(\alpha+1)}{2\left(e^{i \varphi} \eta(b, a)\right)^{\alpha}}\left[J_{a^{+}}^{\alpha} f\left(a+e^{i \varphi} \eta(b, a)\right)+J_{\left(a+e^{i \varphi} \eta(b, a)\right)}^{\alpha} f(a)\right]|,| f^{\prime \prime}(a)|,| f^{\prime \prime}(b) \mid\right) \\
& \quad+\int_{0}^{1} L_{w(t)} d t \leq 0 .
\end{aligned}
$$

Proof Since $\left|f^{\prime \prime}\right|$ is $F$-convex, we can see that

$$
F\left(\left|f^{\prime \prime}\left(a+(1-t) e^{i \varphi} \eta(b, a)\right)\right|,\left|f^{\prime \prime}(a)\right|,\left|f^{\prime \prime}(b)\right|, t\right) \leq 0, \quad t \in[0,1]
$$

Multiplying this inequality by $w(t)=1-(1-t)^{\alpha+1}-t^{\alpha+1}$ and using axiom (A3), we have

$$
F\left(w(t)\left|f^{\prime \prime}\left(a+(1-t) e^{i \varphi} \eta(b, a)\right)\right|, w(t)\left|f^{\prime \prime}(a)\right|, w(t)\left|f^{\prime \prime}(b)\right|, t\right)+L_{w(t)} \leq 0, \quad t \in[0,1]
$$


Integrating over $[0,1]$ and using axiom (A2), we get

$$
\begin{aligned}
& T_{F, w}\left(\int_{0}^{1} w(t)\left|f^{\prime \prime}\left(a+(1-t) e^{i \varphi} \eta(b, a)\right)\right| d t,\left|f^{\prime \prime}(a)\right|,\left|f^{\prime \prime}(b)\right|, t\right)+\int_{0}^{1} L_{w(t)} d t \leq 0, \\
& \quad t \in[0,1] .
\end{aligned}
$$

Using Lemma 2, we have

$$
\begin{aligned}
& \frac{2(\alpha+1)}{\left(e^{i \varphi} \eta(b, a)\right)^{2}} \mid \frac{f(a)+f\left(a+e^{i \varphi} \eta(b, a)\right)}{2} \\
& \quad-\frac{\Gamma(\alpha+1))}{2\left(e^{i \varphi} \eta(b, a)\right)^{\alpha}}\left[J_{a^{+}}^{\alpha} f\left(a+e^{i \varphi} \eta(b, a)\right)+J_{\left(a+e^{i \varphi} \eta(b, a)\right)}^{\alpha} f(a)\right] \mid \\
& \leq \int_{0}^{1}\left[1-(1-t)^{\alpha+1}-t^{\alpha+1}\right]\left|f^{\prime \prime}\left(a+(1-t) e^{i \varphi} \eta(b, a)\right)\right| d t .
\end{aligned}
$$

Because $T_{F, w}$ is nondecreasing with respect to the first variable so that

$$
\begin{aligned}
& T_{F, w}\left(\frac{2(\alpha+1)}{\left(e^{i \varphi} \eta(b, a)\right)^{2}} \mid \frac{f(a)+f\left(a+e^{i \varphi} \eta(b, a)\right)}{2}\right. \\
& \left.-\frac{\Gamma(\alpha+1)}{2\left(e^{i \varphi} \eta(b, a)\right)^{\alpha}}\left[J_{a^{+}}^{\alpha} f\left(a+e^{i \varphi} \eta(b, a)\right)+J_{\left(a+e^{i \varphi} \eta(b, a)\right)}^{\alpha} f(a)\right]|,| f^{\prime \prime}(a)|,| f^{\prime \prime}(b) \mid\right) \\
& \quad+\int_{0}^{1} L_{w(t)} d t \leq 0, \quad t \in[0,1] .
\end{aligned}
$$

This completes the proof.

Remark 11 By taking $\eta(b, a)=b-a$ and $\varphi=0$ in Theorem 12, we obtain

$$
\begin{aligned}
& T_{F, w}\left(\frac{2(\alpha+1)}{(b-a)^{2}}\left|\frac{f(a)+f(b)}{2}-\frac{\Gamma(\alpha+1))}{2(b-a)^{\alpha}}\left[J_{a^{f}}^{\alpha} f(b)+J_{b^{-}}^{\alpha} f(a)\right]\right|,\left|f^{\prime \prime}(a)\right|,\left|f^{\prime \prime}(b)\right|\right) \\
& \quad+\int_{0}^{1} L_{w(t)} d t \leq 0 .
\end{aligned}
$$

Corollary 10 Under the assumptions of Theorem 12, if $\left|f^{\prime \prime}\right|$ is $\varepsilon$-convex, then

$$
\begin{aligned}
& \left|\frac{f(a)+f\left(a+e^{i \varphi} \eta(b, a)\right)}{2}-\frac{\Gamma(\alpha+1))}{2\left(e^{i \varphi} \eta(b, a)\right)^{\alpha}}\left[J_{a^{+}}^{\alpha} f\left(a+e^{i \varphi} \eta(b, a)\right)+J_{\left(a+e^{i \varphi} \eta(b, a)\right)}^{\alpha} f(a)\right]\right| \\
& \quad \leq \frac{\alpha\left(e^{i \varphi} \eta(b, a)\right)^{2}}{4(\alpha+1)(\alpha+2)}\left(\left|f^{\prime \prime}(a)\right|+\left|f^{\prime \prime}(b)\right|+2 \varepsilon\right) .
\end{aligned}
$$

Proof Using (5) with $w(t)=1-(1-t)^{\alpha+1}-t^{\alpha+1}$, we find

$$
\int_{0}^{1} L_{w(t)} d t=\varepsilon \int_{0}^{1}\left((1-t)^{\alpha+1}+t^{\alpha+1}\right) d t=\frac{2 \varepsilon}{\alpha+2}
$$


With $w(t)=1-(1-t)^{\alpha+1}-t^{\alpha+1}$, Eq. (4) gives

$$
\begin{aligned}
T_{F, w}\left(u_{1}, u_{2}, u_{3}\right) \\
=u_{1}-\left(\int_{0}^{1} t\left[1-(1-t)^{\alpha+1}-t^{\alpha+1}\right] d t\right) u_{2} \\
\quad-\left(\int_{0}^{1}(1-t)\left[1-(1-t)^{\alpha+1}-t^{\alpha+1}\right] d t\right) u_{3}-\varepsilon \\
=u_{1}-\frac{\alpha}{2(\alpha+2)}\left(u_{2}+u_{3}\right)-\varepsilon
\end{aligned}
$$

for $u_{1}, u_{2}, u_{3} \in \mathbb{R}$. Hence, by Theorem 12 , we have

$$
\begin{aligned}
0 \geq & T_{F, w}\left(\frac{2(\alpha+1)}{\left(e^{i \varphi} \eta(b, a)\right)^{2}} \mid \frac{f(a)+f\left(a+e^{i \varphi} \eta(b, a)\right)}{2}\right. \\
& \left.-\frac{\Gamma(\alpha+1)}{2\left(e^{i \varphi} \eta(b, a)\right)^{\alpha}}\left[J_{a^{+}}^{\alpha} f\left(a+e^{i \varphi} \eta(b, a)\right)+J_{\left(a+e^{i \varphi} \eta(b, a)\right)}^{\alpha} f(a)\right]|,| f^{\prime \prime}(a)|,| f^{\prime \prime}(b) \mid\right) \\
& +\int_{0}^{1} L_{w(t)} d t \\
= & \frac{2(\alpha+1)}{\left(e^{i \varphi} \eta(b, a)\right)^{2}} \mid \frac{f(a)+f\left(a+e^{i \varphi} \eta(b, a)\right)}{2} \\
& -\frac{\Gamma(\alpha+1)}{2\left(e^{i \varphi} \eta(b, a)\right)^{\alpha}}\left[J_{a^{+}}^{\alpha} f\left(a+e^{i \varphi} \eta(b, a)\right)+J_{\left(a+e^{i \varphi} \eta(b, a)\right)}^{\alpha} f(a)\right] \mid \\
& -\frac{\alpha}{2(\alpha+2)}\left(\left|f^{\prime \prime}(a)\right|+\left|f^{\prime \prime}(b)\right|\right)-\varepsilon+\frac{2 \varepsilon}{\alpha+2} \\
= & \frac{2(\alpha+1)}{\left(e^{i \varphi} \eta(b, a)\right)^{2}} \mid \frac{f(a)+f\left(a+e^{i \varphi} \eta(b, a)\right)}{2} \\
& -\frac{\Gamma(\alpha+1)}{2\left(e^{i \varphi} \eta(b, a)\right)^{\alpha}}\left[J_{a^{+}}^{\alpha} f\left(a+e^{i \varphi} \eta(b, a)\right)+J_{\left(a+e^{i \varphi} \eta(b, a)\right)}^{\alpha} f(a)\right] \mid \\
& -\frac{\alpha}{2(\alpha+2)}\left(\left|f^{\prime \prime}(a)\right|+\left|f^{\prime \prime}(b)\right|\right)-\frac{\alpha}{\alpha+2} \varepsilon .
\end{aligned}
$$

This completes the proof.

Remark 12 In Corollary 10, if we take

(a) $\eta(b, a)=b-a$ and $\varphi=0$, we get

$$
\begin{aligned}
& \left|\frac{f(a)+f(b)}{2}-\frac{\Gamma(\alpha+1))}{2(b-a)^{\alpha}}\left[J_{a^{+}}^{\alpha} f(b)+J_{b^{-}}^{\alpha} f(a)\right]\right| \\
& \quad \leq \frac{\alpha(b-a)^{2}}{4(\alpha+1)(\alpha+2)}\left(\left|f^{\prime \prime}(a)\right|+\left|f^{\prime \prime}(b)\right|+2 \varepsilon\right) .
\end{aligned}
$$

(b) $\eta(b, a)=b-a, \varphi=0$, and $\varepsilon=0$, we get

$$
\left|\frac{f(a)+f(b)}{2}-\frac{\Gamma(\alpha+1))}{2(b-a)^{\alpha}}\left[J_{a^{+}}^{\alpha} f(b)+J_{b^{-}}^{\alpha} f(a)\right]\right| \leq \frac{\alpha(b-a)^{2}}{4(\alpha+1)(\alpha+2)}\left(\left|f^{\prime \prime}(a)\right|+\left|f^{\prime \prime}(b)\right|\right) .
$$


Corollary 11 Under the assumptions of Theorem 12, if $\left|f^{\prime \prime}\right|$ is $\lambda_{\varphi}$-preinvex, then

$$
\begin{aligned}
& \left|\frac{f(a)+f\left(a+e^{i \varphi} \eta(b, a)\right)}{2}-\frac{\Gamma(\alpha+1))}{2\left(e^{i \varphi} \eta(b, a)\right)^{\alpha}}\left[J_{a^{+}}^{\alpha} f\left(a+e^{i \varphi} \eta(b, a)\right)+J_{\left(a+e^{i \varphi} \eta(b, a)\right)^{-}}^{\alpha} f(a)\right]\right| \\
& \quad \leq \frac{\left(e^{i \varphi} \eta(b, a)\right)^{2}}{2(\alpha+1)}\left[\frac{\pi}{2}-\beta\left(\frac{1}{2}, \alpha+\frac{5}{2}\right)-\beta\left(\frac{3}{2}, \alpha+\frac{3}{2}\right)\right]\left(\left|f^{\prime \prime}(a)\right|+\frac{1-\lambda}{\lambda}\left|f^{\prime \prime}(b)\right|\right) .
\end{aligned}
$$

Proof Using (7) with $w(t)=1-(1-t)^{\alpha+1}-t^{\alpha+1}$, we have

$$
\begin{aligned}
T_{F, w}\left(u_{1}, u_{2}, u_{3}\right) \\
=u_{1}-\left(\int_{0}^{1} \frac{\sqrt{t}}{2 \sqrt{1-t}}\left[1-(1-t)^{\alpha+1}-t^{\alpha+1}\right] d t\right) u_{3} \\
\quad-\frac{1-\lambda}{\lambda}\left(\int_{0}^{1} \frac{\sqrt{1-t}}{2 \sqrt{t}}\left[1-(1-t)^{\alpha+1}-t^{\alpha+1}\right] d t\right) u_{2} \\
=u_{1}-\left[\frac{\pi}{2}-\beta\left(\frac{1}{2}, \alpha+\frac{5}{2}\right)-\beta\left(\frac{3}{2}, \alpha+\frac{3}{2}\right)\right]\left(u_{2}+\frac{1-\lambda}{\lambda} u_{3}\right)
\end{aligned}
$$

for $u_{1}, u_{2}, u_{3} \in \mathbb{R}$. Hence, by Theorem 12 , we get

$$
\begin{aligned}
0 \geq & T_{F, w}\left(\frac{2(\alpha+1)}{\left(e^{i \varphi} \eta(b, a)\right)^{2}} \mid \frac{f(a)+f\left(a+e^{i \varphi} \eta(b, a)\right)}{2}\right. \\
& \left.-\frac{\Gamma(\alpha+1)}{2\left(e^{i \varphi} \eta(b, a)\right)^{\alpha}}\left[J_{a^{+}}^{\alpha} f\left(a+e^{i \varphi} \eta(b, a)\right)+J_{\left(a+e^{i \varphi} \eta(b, a)\right)^{\alpha}}^{\alpha} f(a)\right]|,| f^{\prime \prime}(a)|,| f^{\prime \prime}(b) \mid\right) \\
& +\int_{0}^{1} L_{w(t)} d t \\
= & \frac{2(\alpha+1)}{\left(e^{i \varphi} \eta(b, a)\right)^{2}} \mid \frac{f(a)+f\left(a+e^{i \varphi} \eta(b, a)\right)}{2} \\
& -\frac{\Gamma(\alpha+1)}{2\left(e^{i \varphi} \eta(b, a)\right)^{\alpha}}\left[J_{a^{+}}^{\alpha} f\left(a+e^{i \varphi} \eta(b, a)\right)+J_{\left(a+e^{i \varphi} \eta(b, a)\right)^{-}}^{\alpha} f(a)\right] \mid \\
& -\left[\frac{\pi}{2}-\beta\left(\frac{1}{2}, \alpha+\frac{5}{2}\right)-\beta\left(\frac{3}{2}, \alpha+\frac{3}{2}\right)\right]\left(\left|f^{\prime \prime}(a)\right|+\frac{1-\lambda}{\lambda}\left|f^{\prime \prime}(b)\right|\right) .
\end{aligned}
$$

This leads to

$$
\begin{aligned}
& \left|\frac{f(a)+f\left(a+e^{i \varphi} \eta(b, a)\right)}{2}-\frac{\Gamma(\alpha+1)}{2\left(e^{i \varphi} \eta(b, a)\right)^{\alpha}}\left[J_{a^{+}}^{\alpha} f\left(a+e^{i \varphi} \eta(b, a)\right)+J_{\left(a+e^{i \varphi} \eta(b, a)\right)^{-}}^{\alpha} f(a)\right]\right| \\
& \leq \frac{\left(e^{i \varphi} \eta(b, a)\right)^{2}}{2(\alpha+1)}\left[\frac{\pi}{2}-\beta\left(\frac{1}{2}, \alpha+\frac{5}{2}\right)-\beta\left(\frac{3}{2}, \alpha+\frac{3}{2}\right)\right]\left(\left|f^{\prime \prime}(a)\right|+\frac{1-\lambda}{\lambda}\left|f^{\prime \prime}(b)\right|\right) .
\end{aligned}
$$

Thus, the proof is completed.

Remark 13 In Corollary 11, if we choose

(a) $\eta(b, a)=b-a$ and $\varphi=0$, we get

$$
\left|\frac{f(a)+f(b)}{2}-\frac{\Gamma(\alpha+1))}{2(b-a)^{\alpha}}\left[J_{a^{+}}^{\alpha} f(b)+J_{b^{-}}^{\alpha} f(a)\right]\right|
$$




$$
\leq \frac{(b-a)^{2}}{2(\alpha+1)}\left[\frac{\pi}{2}-\beta\left(\frac{1}{2}, \alpha+\frac{5}{2}\right)-\beta\left(\frac{3}{2}, \alpha+\frac{3}{2}\right)\right]\left(\left|f^{\prime \prime}(a)\right|+\frac{1-\lambda}{\lambda}\left|f^{\prime \prime}(b)\right|\right) .
$$

(b) $\eta(b, a)=b-a, \varphi=0$, and $\lambda=\frac{1}{2}$, we get

$$
\begin{aligned}
& \left|\frac{f(a)+f(b)}{2}-\frac{\Gamma(\alpha+1))}{2(b-a)^{\alpha}}\left[J_{a^{+}}^{\alpha} f(b)+J_{b^{-}}^{\alpha} f(a)\right]\right| \\
& \quad \leq \frac{(b-a)^{2}}{2(\alpha+1)}\left[\frac{\pi}{2}-\beta\left(\frac{1}{2}, \alpha+\frac{5}{2}\right)-\beta\left(\frac{3}{2}, \alpha+\frac{3}{2}\right)\right]\left(\left|f^{\prime \prime}(a)\right|+\left|f^{\prime \prime}(b)\right|\right) .
\end{aligned}
$$

Corollary 12 Under the assumptions of Theorem 12, if $\left|f^{\prime \prime}\right|$ is h-convex, then we have

$$
\begin{gathered}
\left|\frac{f(a)+f\left(a+e^{i \varphi} \eta(b, a)\right)}{2}-\frac{\Gamma(\alpha+1))}{2\left(e^{i \varphi} \eta(b, a)\right)^{\alpha}}\left[J_{a^{+}}^{\alpha} f\left(a+e^{i \varphi} \eta(b, a)\right)+J_{\left(a+e^{i \varphi} \eta(b, a)\right)}^{\alpha} f(a)\right]\right| \\
\quad \leq \frac{\left(e^{i \varphi} \eta(b, a)\right)^{2}}{2(\alpha+1)}\left(\int_{0}^{1} h(t)\left[1-(1-t)^{\alpha+1}-t^{\alpha+1}\right] d t\right)\left(\left|f^{\prime \prime}(a)\right|+\frac{1-\lambda}{\lambda}\left|f^{\prime \prime}(b)\right|\right) .
\end{gathered}
$$

Proof Using (9) with $w(t)=1-(1-t)^{\alpha+1}-t^{\alpha+1}$, we obtain

$$
\begin{aligned}
T_{F, w}\left(u_{1}, u_{2}, u_{3}\right) \\
=u_{1}-\left(\int_{0}^{1} h(t)\left[1-(1-t)^{\alpha+1}-t^{\alpha+1}\right] d t\right) u_{3} \\
\quad-\frac{1-\lambda}{\lambda}\left(\int_{0}^{1} h(1-t)\left[1-(1-t)^{\alpha+1}-t^{\alpha+1}\right] d t\right) u_{2} \\
=u_{1}-\left(\int_{0}^{1} h(t)\left[1-(1-t)^{\alpha+1}-t^{\alpha+1}\right] d t\right) u_{3} \\
\quad-\frac{1-\lambda}{\lambda}\left(\int_{0}^{1} h(t)\left[1-(1-t)^{\alpha+1}-t^{\alpha+1}\right] d t\right) u_{2} \\
=u_{1}-\left(\int_{0}^{1} h(t)\left[1-(1-t)^{\alpha+1}-t^{\alpha+1}\right] d t\right)\left(u_{2}+\frac{1-\lambda}{\lambda} u_{3}\right)
\end{aligned}
$$

for $u_{1}, u_{2}, u_{3} \in \mathbb{R}$, so Theorem 12 implies that

$$
\begin{aligned}
0 \geq & T_{F, w}\left(\frac{2(\alpha+1)}{\left(e^{i \varphi} \eta(b, a)\right)^{2}} \mid \frac{f(a)+f\left(a+e^{i \varphi} \eta(b, a)\right)}{2}\right. \\
& \left.-\frac{\Gamma(\alpha+1)}{2\left(e^{i \varphi} \eta(b, a)\right)^{\alpha}}\left[J_{a^{+}}^{\alpha} f\left(a+e^{i \varphi} \eta(b, a)\right)+J_{\left(a+e^{i \varphi} \eta(b, a)\right)}^{\alpha} f(a)\right]|,| f^{\prime \prime}(a)|,| f^{\prime \prime}(b) \mid\right) \\
& +\int_{0}^{1} L_{w(t)} d t \\
= & \frac{2(\alpha+1)}{\left(e^{i \varphi} \eta(b, a)\right)^{2}} \mid \frac{f(a)+f\left(a+e^{i \varphi} \eta(b, a)\right)}{2} \\
& -\frac{\Gamma(\alpha+1)}{2\left(e^{i \varphi} \eta(b, a)\right)^{\alpha}}\left[J_{a^{\alpha}}^{\alpha} f\left(a+e^{i \varphi} \eta(b, a)\right)+J_{\left(a+e^{i \varphi} \eta(b, a)\right)}^{\alpha} f(a)\right] \mid \\
& -\left(\int_{0}^{1} h(t)\left[1-(1-t)^{\alpha+1}-t^{\alpha+1}\right] d t\right)\left(\left|f^{\prime \prime}(a)\right|+\frac{1-\lambda}{\lambda}\left|f^{\prime \prime}(b)\right|\right),
\end{aligned}
$$


which can be written as

$$
\begin{gathered}
\left|\frac{f(a)+f\left(a+e^{i \varphi} \eta(b, a)\right)}{2}-\frac{\Gamma(\alpha+1)}{2\left(e^{i \varphi} \eta(b, a)\right)^{\alpha}}\left[J_{a^{+}}^{\alpha} f\left(a+e^{i \varphi} \eta(b, a)\right)+J_{\left(a+e^{i \varphi} \eta(b, a)\right)}^{\alpha} f(a)\right]\right| \\
\leq \frac{\left(e^{i \varphi} \eta(b, a)\right)^{2}}{2(\alpha+1)}\left(\int_{0}^{1} h(t)\left[1-(1-t)^{\alpha+1}-t^{\alpha+1}\right] d t\right)\left(\left|f^{\prime \prime}(a)\right|+\frac{1-\lambda}{\lambda}\left|f^{\prime \prime}(b)\right|\right) .
\end{gathered}
$$

Thus, the proof is done.

Theorem 13 Let $f:[0, b] \rightarrow \mathbb{R}$ be a differentiable mapping and $\left.\left.\right|^{\prime \prime}\right|^{\frac{p}{p-1}}$ is measurable, decreasing, $\lambda_{\varphi}$-preinvex function on $[0, b]$ for $\eta(b, a)>0$ and $0 \leq a<b$. Suppose that $\left|f^{\prime \prime}\right| \frac{p}{p-1}$ is $F$-convex on $[a, b]$, for some $F \in \mathcal{F}$ and $\left|f^{\prime \prime}\right| \in L^{\frac{p}{p-1}}(a, b), p>1$. Then we have

$$
T_{F, 1}\left(H_{1}(f, p),\left|f^{\prime \prime}(a)\right|^{\frac{p}{p-1}},\left|f^{\prime \prime}(b)\right|^{\frac{p}{p-1}}\right) \leq 0,
$$

where

$$
\begin{aligned}
H_{1}(f, p)= & \left(\frac{2^{\alpha+1}(\alpha+1)}{\left(2^{\alpha}-1\right)\left(e^{i \varphi} \eta(b, a)\right)^{2}}\right)^{\frac{p}{p-1}} \mid \frac{f(a)+f\left(a+e^{i \varphi} \eta(b, a)\right)}{2} \\
& -\frac{\Gamma(\alpha+1)}{2\left(e^{i \varphi} \eta(b, a)\right)^{\alpha}}\left[J_{a^{+}}^{\alpha} f\left(a+e^{i \varphi} \eta(b, a)\right)+J_{\left(a+e^{i \varphi} \eta(b, a)\right)}^{\alpha} f(a)\right] \mid
\end{aligned}
$$

Proof Since $\left|f^{\prime \prime}\right|^{\frac{p}{p-1}}$ is $F$-convex, we have

$$
F\left(\left|f^{\prime \prime}\left(a+(1-t) e^{i \varphi} \eta(b, a)\right)\right|^{\frac{p}{p-1}},\left|f^{\prime \prime}(a)\right|^{\frac{p}{p-1}},\left|f^{\prime \prime}(b)\right|^{\frac{p}{p-1}}, t\right) \leq 0, \quad t \in[0,1] .
$$

Using (A2) with $w(t)=1$, we have

$$
T_{F, 1}\left(\int_{0}^{1}\left|f\left(a+(1-t) e^{i \varphi} \eta(b, a)\right)\right|^{\frac{p}{p-1}} d t,\left|f^{\prime \prime}(a)\right|^{\frac{p}{p-1}},\left|f^{\prime \prime}(b)\right|^{\frac{p}{p-1}}\right) \leq 0, \quad t \in[0,1] .
$$

Using Lemma 2, Lemma 3 and the Hölder inequality, we get

$$
\begin{aligned}
& \left|\frac{f(a)+f\left(a+e^{i \varphi} \eta(b, a)\right)}{2}-\frac{\Gamma(\alpha+1)}{2\left(e^{i \varphi} \eta(b, a)\right)^{\alpha}}\left[J_{a^{+}}^{\alpha} f\left(a+e^{i \varphi} \eta(b, a)\right)+J_{\left(a+e^{i \varphi} \eta(b, a)\right)}^{\alpha} f(a)\right]\right| \\
& =\frac{\left(e^{i \varphi} \eta(b, a)\right)^{2}}{2(\alpha+1)} \int_{0}^{1}\left[1-(1-t)^{\alpha+1}-t^{\alpha+1}\right]\left|f^{\prime \prime}\left(a+e^{i \varphi} \eta(b, a)\right)\right| d t \\
& \leq \frac{\left(e^{i \varphi} \eta(b, a)\right)^{2}}{2(\alpha+1)}\left(\int_{0}^{1}\left[1-(1-t)^{\alpha+1}-t^{\alpha+1}\right]^{p} d t\right)^{\frac{1}{p}} \\
& \quad \times\left(\int_{0}^{1}\left|f^{\prime \prime}\left(a+(1-t) e^{i \varphi} \eta(b, a)\right)\right|^{\frac{p}{p-1}} d t\right)^{\frac{p-1}{p}} \\
& =\frac{\left(e^{i \varphi} \eta(b, a)\right)^{2}}{2(\alpha+1)}\left(1-\frac{1}{2^{\alpha}}\right)\left(\int_{0}^{1}\left|f^{\prime \prime}\left(a+(1-t) e^{i \varphi} \eta(b, a)\right)\right|^{\frac{p}{p-1}} d t\right)^{\frac{p-1}{p}}
\end{aligned}
$$

or, equivalently,

$$
\left(\frac{2^{\alpha+1}(\alpha+1)}{\left(2^{\alpha}-1\right)\left(e^{i \varphi} \eta(b, a)\right)^{2}}\right)^{\frac{p}{p-1}} \mid \frac{f(a)+f\left(a+e^{i \varphi} \eta(b, a)\right)}{2}
$$




$$
\begin{aligned}
& -\left.\frac{\Gamma(\alpha+1)}{2\left(e^{i \varphi} \eta(b, a)\right)^{\alpha}}\left[J_{a^{+}}^{\alpha} f\left(a+e^{i \varphi} \eta(b, a)\right)+J_{\left(a+e^{i \varphi} \eta(b, a)\right)}^{\alpha} f(a)\right]\right|^{\frac{p}{p-1}} \\
\leq & \int_{0}^{1}\left|f^{\prime \prime}\left(a+(1-t) e^{i \varphi} \eta(b, a)\right)\right|^{\frac{p}{p-1}} d t .
\end{aligned}
$$

Because $T_{F, 1}$ is nondecreasing with respect to the first variable, we get

$$
T_{F, 1}\left(H_{1}(f, p),\left|f^{\prime \prime}(a)\right|^{\frac{p}{p-1}},\left|f^{\prime \prime}(b)\right|^{\frac{p}{p-1}}\right) \leq 0 .
$$

Thus, the proof is completed.

Remark 14 If we choose $\eta(b, a)=b-a$ and $\varphi=0$ in Theorem 13, we get

$$
\begin{aligned}
& T_{F, 1}\left(\left(\frac{2^{\alpha+1}(\alpha+1)}{\left(2^{\alpha}-1\right)(b-a)^{2}}\right)^{\frac{p}{p-1}}\left|\frac{f(a)+f(b)}{2}-\frac{\Gamma(\alpha+1))}{2(b-a)^{\alpha}}\left[J_{a^{+}}^{\alpha} f(b)+J_{b^{-}}^{\alpha} f(a)\right]\right|,\right. \\
& \left.\quad f^{\prime \prime}(a)|,| f^{\prime \prime}(b) \mid\right) \leq 0 .
\end{aligned}
$$

Corollary 13 Under the assumptions of Theorem 13, if $\left|f^{\prime \prime}\right|^{\frac{p}{p-1}}$ is $\varepsilon$-convex, then

$$
\begin{aligned}
& \left|\frac{f(a)+f\left(a+e^{i \varphi} \eta(b, a)\right)}{2}-\frac{\Gamma(\alpha+1))}{2\left(e^{i \varphi} \eta(b, a)\right)^{\alpha}}\left[J_{a^{+}}^{\alpha} f\left(a+e^{i \varphi} \eta(b, a)\right)+J_{\left(a+e^{i \varphi} \eta(b, a)\right)}^{\alpha} f(a)\right]\right| \\
& \leq\left(\frac{\left(2^{\alpha}-1\right)\left(e^{i \varphi} \eta(b, a)\right)^{2}}{2^{\alpha+1}(\alpha+1)}\right)\left(\frac{\left|f^{\prime \prime}(a)\right|^{\frac{p}{p-1}}+\left|f^{\prime \prime}(b)\right|^{\frac{p}{p-1}}}{2}+\varepsilon\right)^{\frac{p-1}{p}} .
\end{aligned}
$$

Proof Using (12), (13), by Theorem 13, we have

$$
\begin{aligned}
0 & \geq T_{F, 1}\left(H_{1}(f, p),\left|f^{\prime \prime}(a)\right|^{\frac{p}{p-1}},\left|f^{\prime \prime}(b)\right|^{\frac{p}{p-1}}\right) \\
& =H_{1}(f, p)-\frac{\left|f^{\prime \prime}(a)\right|^{\frac{p}{p-1}}+\left|f^{\prime \prime}(b)\right|^{\frac{p}{p-1}}}{2}-\varepsilon .
\end{aligned}
$$

This leads to

$$
\begin{aligned}
& \left|\frac{f(a)+f\left(a+e^{i \varphi} \eta(b, a)\right)}{2}-\frac{\Gamma(\alpha+1))}{2\left(e^{i \varphi} \eta(b, a)\right)^{\alpha}}\left[J_{a^{+}}^{\alpha} f\left(a+e^{i \varphi} \eta(b, a)\right)+J_{\left(a+e^{i \varphi} \eta(b, a)\right)}^{\alpha} f(a)\right]\right| \\
& \quad \leq\left(\frac{\left(2^{\alpha}-1\right)\left(e^{i \varphi} \eta(b, a)\right)^{2}}{2^{\alpha+1}(\alpha+1)}\right)\left(\frac{\left|f^{\prime \prime}(a)\right|^{\frac{p}{p-1}}+\left|f^{\prime \prime}(b)\right|^{\frac{p}{p-1}}}{2}+\varepsilon\right)^{\frac{p-1}{p}} .
\end{aligned}
$$

This completes the proof.

Remark 15 In Corollary 13, if we choose

(a) $\eta(b, a)=b-a$ and $\varphi=0$, we get

$$
\begin{aligned}
& \left|\frac{f(a)+f(b)}{2}-\frac{\Gamma(\alpha+1))}{2(b-a)^{\alpha}}\left[J_{a^{+}}^{\alpha} f(b)+J_{b^{-}}^{\alpha} f(a)\right]\right| \\
& \quad \leq\left(\frac{\left(2^{\alpha}-1\right)(b-a)^{2}}{2^{\alpha+1}(\alpha+1)}\right)\left(\frac{\left|f^{\prime \prime}(a)\right|^{\frac{p}{p-1}}+\left|f^{\prime \prime}(b)\right|^{\frac{p}{p-1}}}{2}+\varepsilon\right)^{\frac{p-1}{p}} .
\end{aligned}
$$


(b) $\eta(b, a)=b-a, \varphi=0$, and $\varepsilon=0$, we get

$$
\begin{aligned}
& \left|\frac{f(a)+f(b)}{2}-\frac{\Gamma(\alpha+1))}{2(b-a)^{\alpha}}\left[J_{a^{+}}^{\alpha} f(b)+J_{b^{-}}^{\alpha} f(a)\right]\right| \\
& \quad \leq\left(\frac{\left(2^{\alpha}-1\right)(b-a)^{2}}{2^{\alpha+1}(\alpha+1)}\right)\left(\frac{\left|f^{\prime \prime}(a)\right|^{\frac{p}{p-1}}+\left|f^{\prime \prime}(b)\right|^{\frac{p}{p-1}}}{2}\right)^{\frac{p-1}{p}} .
\end{aligned}
$$

Corollary 14 Under the assumptions of Theorem 13. If $\left|f^{\prime \prime}\right|^{\frac{p-1}{p}}$ is $\lambda_{\varphi}$-preinvex, we have

$$
\begin{aligned}
& \left|\frac{f(a)+f\left(a+e^{i \varphi} \eta(b, a)\right)}{2}-\frac{\Gamma(\alpha+1))}{2\left(e^{i \varphi} \eta(b, a)\right)^{\alpha}}\left[J_{a^{+}}^{\alpha} f\left(a+e^{i \varphi} \eta(b, a)\right)+J_{\left(a+e^{i \varphi} \eta(b, a)\right)}^{\alpha} f(a)\right]\right| \\
& \leq\left(\frac{\left(2^{\alpha}-1\right)\left(e^{i \varphi} \eta(b, a)\right)^{2}}{2^{\alpha+1}(\alpha+1)}\right)\left[\frac{\pi}{4}\left(\left|f^{\prime \prime}(a)\right|^{\frac{p-1}{p}}+\frac{1-\lambda}{\lambda}\left|f^{\prime \prime}(b)\right|^{\frac{p-1}{p}}\right)\right]^{\frac{p-1}{p}} .
\end{aligned}
$$

Proof Using (7), (14), by Theorem 13, we have

$$
\begin{aligned}
0 \geq & T_{F, 1}\left(H_{1}(f, p),\left|f^{\prime \prime}(a)\right|^{\frac{p-1}{p}},\left|f^{\prime \prime}(b)\right|^{\frac{p-1}{p}}\right) \\
= & \left(\frac{2^{\alpha+1}(\alpha+1)}{\left(2^{\alpha}-1\right)\left(e^{i \varphi} \eta(b, a)\right)^{2}}\right)^{\frac{p}{p-1}} \mid \frac{f(a)+f\left(a+e^{i \varphi} \eta(b, a)\right)}{2} \\
& -\left.\frac{\Gamma(\alpha+1)}{2\left(e^{i \varphi} \eta(b, a)\right)^{\alpha}}\left[J_{a^{+}}^{\alpha} f\left(a+e^{i \varphi} \eta(b, a)\right)+J_{\left(a+e^{i \varphi} \eta(b, a)\right)}^{\alpha} f(a)\right]\right|^{\frac{p}{p-1}} \\
& -\frac{\pi}{4}\left(\left|f^{\prime \prime}(a)\right|^{\frac{p-1}{p}}+\frac{1-\lambda}{\lambda}\left|f^{\prime \prime}(b)\right|^{\frac{p-1}{p}}\right),
\end{aligned}
$$

that is,

$$
\begin{aligned}
& \left|\frac{f(a)+f\left(a+e^{i \varphi} \eta(b, a)\right)}{2}-\frac{\Gamma(\alpha+1)}{2\left(e^{i \varphi} \eta(b, a)\right)^{\alpha}}\left[J_{a^{+}}^{\alpha} f\left(a+e^{i \varphi} \eta(b, a)\right)+J_{\left(a+e^{i \varphi} \eta(b, a)\right)}^{\alpha} f(a)\right]\right| \\
& \leq\left(\frac{\left(2^{\alpha}-1\right)\left(e^{i \varphi} \eta(b, a)\right)^{2}}{2^{\alpha+1}(\alpha+1)}\right)\left[\frac{\pi}{4}\left(\left|f^{\prime \prime}(a)\right|^{\frac{p-1}{p}}+\frac{1-\lambda}{\lambda}\left|f^{\prime \prime}(b)\right|^{\frac{p-1}{p}}\right)\right]^{\frac{p-1}{p}} .
\end{aligned}
$$

This proves Corollary 14.

Remark 16 In Corollary 14, if we choose

(a) $\eta(b, a)=b-a$ and $\varphi=0$, we get

$$
\begin{aligned}
& \left|\frac{f(a)+f(b)}{2}-\frac{\Gamma(\alpha+1))}{2(b-a)^{\alpha}}\left[J_{a^{+}}^{\alpha} f(b)+J_{b^{-}}^{\alpha} f(a)\right]\right| \\
& \quad \leq\left(\frac{\left(2^{\alpha}-1\right)(b-a)^{2}}{2^{\alpha+1}(\alpha+1)}\right)\left[\frac{\pi}{4}\left(\left|f^{\prime \prime}(a)\right|^{\frac{p-1}{p}}+\frac{1-\lambda}{\lambda}\left|f^{\prime \prime}(b)\right|^{\frac{p-1}{p}}\right)\right]^{\frac{p-1}{p}} .
\end{aligned}
$$


(b) $\eta(b, a)=b-a, \varphi=0$, and $\lambda=\frac{1}{2}$, we get

$$
\begin{aligned}
& \left|\frac{f(a)+f(b)}{2}-\frac{\Gamma(\alpha+1))}{2(b-a)^{\alpha}}\left[J_{a^{+}}^{\alpha} f(b)+J_{b^{-}}^{\alpha} f(a)\right]\right| \\
& \quad \leq\left(\frac{\left(2^{\alpha}-1\right)(b-a)^{2}}{2^{\alpha+1}(\alpha+1)}\right)\left[\frac{\pi\left|f^{\prime \prime}(a)\right|^{\frac{p-1}{p}}+\pi\left|f^{\prime \prime}(b)\right|^{\frac{p-1}{p}}}{4}\right]^{\frac{p-1}{p}} .
\end{aligned}
$$

Corollary 15 Under the assumptions of Theorem 13. If $\left|f^{\prime \prime}\right|^{\frac{p}{p-1}}$ is h-convex, then

$$
\begin{aligned}
& \left|\frac{f(a)+f\left(a+e^{i \varphi} \eta(b, a)\right)}{2}-\frac{\Gamma(\alpha+1))}{2\left(e^{i \varphi} \eta(b, a)\right)^{\alpha}}\left[J_{a^{+}}^{\alpha} f\left(a+e^{i \varphi} \eta(b, a)\right)+J_{\left(a+e^{i \varphi} \eta(b, a)\right)-}^{\alpha} f(a)\right]\right| \\
& \leq\left(\frac{\left(2^{\alpha}-1\right)\left(e^{i \varphi} \eta(b, a)\right)^{2}}{2^{\alpha+1}(\alpha+1)}\right)\left(\int_{0}^{1} h(t)\right)^{\frac{p-1}{p}}\left(\left|f^{\prime \prime}(a)\right|^{\frac{p-1}{p}}+\frac{1-\lambda}{\lambda}\left|f^{\prime \prime}(b)\right|^{\frac{p-1}{p}}\right) .
\end{aligned}
$$

Proof Using (9) and by Theorem 13, it can be proved easily. It is omitted.

Theorem 14 Let $f:[0, b] \rightarrow \mathbb{R}$ be a differentiable mapping and $\left|f^{\prime \prime}\right|^{\frac{p}{p-1}}$ is measurable, decreasing, $\lambda_{\varphi}$-preinvex function on $[0, b]$ for $\eta(b, a)>0$ and $0 \leq a<b$. Suppose that $\left|f^{\prime \prime}\right|^{\frac{p}{p-1}}$ is $F$-convex on $[a, b]$, for some $F \in \mathcal{F}$ and $\left|f^{\prime \prime}\right| \in L^{\frac{p}{p-1}}(a, b), p>1$. Then we have

$$
T_{F, 1}\left(H_{2}(f, p),\left|f^{\prime \prime}(a)\right|^{\frac{p}{p-1}},\left|f^{\prime \prime}(b)\right|^{\frac{p}{p-1}}\right)+\int_{0}^{1} L_{w(t)} d t \leq 0,
$$

where

$$
\begin{aligned}
H_{2}(f, p)= & \left(\frac{2(\alpha+1)}{\left(e^{i \varphi} \eta(b, a)\right)^{2}}\right)^{\frac{p}{p-1}}\left(\frac{2^{\alpha}}{2^{\alpha}-1}\right)^{\frac{1}{p-1}} \mid \frac{f(a)+f\left(a+e^{i \varphi} \eta(b, a)\right)}{2} \\
& -\left.\frac{\Gamma(\alpha+1)}{2\left(e^{i \varphi} \eta(b, a)\right)^{\alpha}}\left[J_{a^{+}}^{\alpha} f\left(a+e^{i \varphi} \eta(b, a)\right)+J_{\left(a+e^{i \varphi} \eta(b, a)\right)}^{\alpha} f(a)\right]\right|^{\frac{p}{p-1}}
\end{aligned}
$$

for $w(t)=1-(1-t)^{\alpha+1}-t^{\alpha+1}$.

Proof Since $\left|f^{\prime \prime}\right|^{\frac{p}{p-1}}$ is $F$-convex, we have

$$
F\left(\left|f^{\prime \prime}\left(a+(1-t) e^{i \varphi} \eta(b, a)\right)\right|^{\frac{p}{p-1}},\left|f^{\prime \prime}(a)\right|^{\frac{p}{p-1}},\left|f^{\prime \prime}(b)\right|^{\frac{p}{p-1}}, t\right) \leq 0, \quad t \in[0,1] .
$$

Using (A3) with $w(t)=1-(1-t)^{\alpha+1}-t^{\alpha+1}$, we obtain

$$
\begin{aligned}
& F\left(w(t)\left|f^{\prime \prime}\left(a+(1-t) e^{i \varphi} \eta(b, a)\right)\right|^{\frac{p}{p-1}}, w(t)\left|f^{\prime \prime}(a)\right|^{\frac{p}{p-1}}, w(t)\left|f^{\prime \prime}(b)\right|^{\frac{p}{p-1}}, t\right)+L_{w(t)} \leq 0, \\
& \quad t \in[0,1] .
\end{aligned}
$$

Integrating over $[0,1]$ and using axiom (A2), we obtain

$$
\begin{aligned}
& T_{F, w}\left(\int_{0}^{1} w(t)\left|f^{\prime \prime}\left(a+(1-t) e^{i \varphi} \eta(b, a)\right)\right|^{\frac{p}{p-1}} d t,\left|f^{\prime \prime}(a)\right|^{\frac{p}{p-1}},\left|f^{\prime \prime}(b)\right|^{\frac{p}{p-1}}\right)+\int_{0}^{1} L_{w(t)} d t \leq 0, \\
& \quad t \in[0,1] .
\end{aligned}
$$


Using Lemma 2 and the power mean inequality, we get

$$
\begin{aligned}
& \left|\frac{f(a)+f\left(a+e^{i \varphi} \eta(b, a)\right)}{2}-\frac{\Gamma(\alpha+1)}{2\left(e^{i \varphi} \eta(b, a)\right)^{\alpha}}\left[J_{a^{+}}^{\alpha} f\left(a+e^{i \varphi} \eta(b, a)\right)+J_{\left(a+e^{i \varphi} \eta(b, a)\right)^{-}}^{\alpha} f(a)\right]\right| \\
& =\frac{e^{i \varphi} \eta(b, a)}{2} \int_{0}^{1}\left[1-(1-t)^{\alpha+1}-t^{\alpha+1}\right]\left|f^{\prime \prime}\left(a+(1-t) e^{i \varphi} \eta(b, a)\right)\right| d t \\
& \leq \frac{\left(e^{i \varphi} \eta(b, a)\right)^{2}}{2(\alpha+1)}\left(\int_{0}^{1}\left[1-(1-t)^{\alpha+1}-t^{\alpha+1}\right] d t\right)^{\frac{1}{p}} \\
& \quad \times\left(\int_{0}^{1} w(t)\left|f^{\prime}\left(a+(1-t) e^{i \varphi} \eta(b, a)\right)\right|^{\frac{p}{p-1}} d t\right)^{\frac{p-1}{p}} \\
& =\frac{\left(e^{i \varphi} \eta(b, a)\right)^{2}}{2(\alpha+1)}\left(1-\frac{1}{2^{\alpha}}\right)^{\frac{1}{p}}\left(\int_{0}^{1} w(t)\left|f^{\prime}\left(a+(1-t) e^{i \varphi} \eta(b, a)\right)\right|^{\frac{p}{p-1}} d t\right)^{\frac{p-1}{p}}
\end{aligned}
$$

or, equivalently,

$$
\begin{aligned}
& \left(\frac{2(\alpha+1)}{\left(e^{i \varphi} \eta(b, a)\right)^{2}}\right)^{\frac{p}{p-1}}\left(\frac{2^{\alpha}}{2^{\alpha}-1}\right)^{\frac{1}{p-1}} \mid \frac{f(a)+f\left(a+e^{i \varphi} \eta(b, a)\right)}{2} \\
& \quad-\left.\frac{\Gamma(\alpha+1)}{2\left(e^{i \varphi} \eta(b, a)\right)^{\alpha}}\left[J_{a^{+}}^{\alpha} f\left(a+e^{i \varphi} \eta(b, a)\right)+J_{\left(a+e^{i \varphi} \eta(b, a)\right)^{-}}^{\alpha} f(a)\right]\right|^{\frac{p}{p-1}} \\
& \leq \int_{0}^{1} w(t)\left|f^{\prime \prime}\left(a+(1-t) e^{i \varphi} \eta(b, a)\right)\right|^{\frac{p}{p-1}} d t .
\end{aligned}
$$

Since $T_{F, w}$ is nondecreasing with respect to the first variable, we have

$$
T_{F, w}\left(H_{2}(f, p),\left|f^{\prime \prime}(a)\right|^{\frac{p}{p-1}},\left|f^{\prime \prime}(b)\right|^{\frac{p}{p-1}}\right)+\int_{0}^{1} L_{w(t)} d t \leq 0 .
$$

This completes the proof.

Remark 17 Taking $\eta(b, a)=b-a$ and $\varphi=0$ in Theorem 14 , we get

$$
\begin{aligned}
& T_{F, w}\left(\left(\frac{2(\alpha+1)}{\left(e^{i \varphi} \eta(b, a)\right)^{2}}\right)^{\frac{p}{p-1}}\left(\frac{2^{\alpha}}{2^{\alpha}-1}\right)^{\frac{1}{p-1}}\left|\frac{f(a)+f(b)}{2}-\frac{\Gamma(\alpha+1))}{2(b-a)^{\alpha}}\left[J_{a^{+}}^{\alpha} f(b)+J_{b^{-}}^{\alpha} f(a)\right]\right|\right. \\
& \left.\left|f^{\prime}(a)\right|,\left|f^{\prime}(b)\right|\right)+\int_{0}^{1} L_{w(t)} d t \leq 0 .
\end{aligned}
$$

Corollary 16 Under the assumptions of Theorem 14, if $\left|f^{\prime \prime}\right|^{\frac{p}{p-1}}$ is $\varepsilon$-convex, we have

$$
\begin{aligned}
& \left|\frac{f(a)+f\left(a+e^{i \varphi} \eta(b, a)\right)}{2}-\frac{\Gamma(\alpha+1))}{2\left(e^{i \varphi} \eta(b, a)\right)^{\alpha}}\left[J_{a^{+}}^{\alpha} f\left(a+e^{i \varphi} \eta(b, a)\right)+J_{\left(a+e^{i \varphi} \eta(b, a)\right)^{-}}^{\alpha} f(a)\right]\right| \\
& \leq\left(\frac{\left(e^{i \varphi} \eta(b, a)\right)^{2}}{2(\alpha+1)}\right)\left(\frac{2^{\alpha}-1}{2^{\alpha}}\right)^{\frac{1}{p}}\left[\frac{\alpha}{2(\alpha+2)}\left(\left|f^{\prime \prime}(a)\right|^{\frac{p}{p-1}}+\left|f^{\prime \prime}(b)\right|^{\frac{p}{p-1}}\right)+2 \varepsilon\right]^{\frac{p-1}{p}} .
\end{aligned}
$$


Proof Using (17), (18) and by Theorem 13, we obtain

$$
\begin{gathered}
\left|\frac{f(a)+f\left(a+e^{i \varphi} \eta(b, a)\right)}{2}-\frac{\Gamma(\alpha+1))}{2\left(e^{i \varphi} \eta(b, a)\right)^{\alpha}}\left[J_{a^{+}}^{\alpha} f\left(a+e^{i \varphi} \eta(b, a)\right)+J_{\left(a+e^{i \varphi} \eta(b, a)\right)}^{\alpha} f(a)\right]\right| \\
\leq\left(\frac{\left(e^{i \varphi} \eta(b, a)\right)^{2}}{2(\alpha+1)}\right)\left(\frac{2^{\alpha}-1}{2^{\alpha}}\right)^{\frac{1}{p}}\left[\frac{\alpha}{2(\alpha+2)}\left(\left|f^{\prime \prime}(a)\right|^{\frac{p}{p-1}}+\left|f^{\prime \prime}(b)\right|^{\frac{p}{p-1}}\right)+2 \varepsilon\right]^{\frac{p-1}{p}} .
\end{gathered}
$$

This completes the proof.

Remark 18 In Corollary 16, if we choose

(a) $\eta(b, a)=b-a$ and $\varphi=0$, we get

$$
\begin{aligned}
& \left|\frac{f(a)+f(b)}{2}-\frac{\Gamma(\alpha+1))}{2(b-a)^{\alpha}}\left[J_{a^{+}}^{\alpha} f(b)+J_{b}^{\alpha} f(a)\right]\right| \\
& \quad \leq\left(\frac{(b-a)^{2}}{2(\alpha+1)}\right)\left(\frac{2^{\alpha}-1}{2^{\alpha}}\right)^{\frac{1}{p}}\left[\frac{\alpha}{2(\alpha+2)}\left(\left|f^{\prime \prime}(a)\right|^{\frac{p}{p-1}}+\left|f^{\prime \prime}(b)\right|^{\frac{p}{p-1}}\right)+2 \varepsilon\right]^{\frac{p-1}{p}} .
\end{aligned}
$$

(b) $\eta(b, a)=b-a, \varphi=0$, and $\varepsilon=0$, we get

$$
\begin{aligned}
& \left|\frac{f(a)+f(b)}{2}-\frac{\Gamma(\alpha+1))}{2(b-a)^{\alpha}}\left[J_{a^{+}}^{\alpha} f(b)+J_{b^{-}}^{\alpha} f(a)\right]\right| \\
& \quad \leq\left(\frac{(b-a)^{2}}{2(\alpha+1)}\right)\left(\frac{2^{\alpha}-1}{2^{\alpha}}\right)^{\frac{1}{p}}\left[\frac{\alpha}{2(\alpha+2)}\left(\left|f^{\prime \prime}(a)\right|^{\frac{p}{p-1}}+\left|f^{\prime \prime}(b)\right|^{\frac{p}{p-1}}\right)\right]^{\frac{p-1}{p}} .
\end{aligned}
$$

Corollary 17 Under the assumptions of Theorem 14. If $\left|f^{\prime \prime}\right|^{\frac{p-1}{p}}$ is $\lambda_{\varphi}$-preinvex, then

$$
\begin{aligned}
& \left|\frac{f(a)+f\left(a+e^{i \varphi} \eta(b, a)\right)}{2}-\frac{\Gamma(\alpha+1))}{2\left(e^{i \varphi} \eta(b, a)\right)^{\alpha}}\left[J_{a^{f}}^{\alpha} f\left(a+e^{i \varphi} \eta(b, a)\right)+J_{\left(a+e^{i \varphi} \eta(b, a)\right)}^{\alpha} f(a)\right]\right| \\
& \leq\left(\frac{\left(e^{i \varphi} \eta(b, a)\right)^{2}}{2(\alpha+1)}\right)\left(\frac{2^{\alpha}-1}{2^{\alpha}}\right)^{\frac{1}{p}} \\
& \quad \times\left(\left[\frac{\pi}{2}-\beta\left(\frac{1}{2}, \alpha+\frac{5}{2}\right)-\beta\left(\frac{3}{2}, \alpha+\frac{3}{2}\right)\right]\left(\left|f^{\prime \prime}(a)\right|^{\frac{p}{p-1}}+\frac{1-\lambda}{\lambda}\left|f^{\prime \prime}(b)\right|^{\frac{p}{p-1}}\right)\right)^{\frac{p-1}{p}} .
\end{aligned}
$$

Proof Using (19), by Theorem 14, we have

$$
\begin{aligned}
0 \geq & T_{F, w}\left(H_{2}(f, p),\left|f^{\prime \prime}(a)\right|^{\frac{p-1}{p}},\left|f^{\prime \prime}(b)\right|^{\frac{p-1}{p}}\right) \\
= & \left(\frac{2(\alpha+1)}{\left(e^{i \varphi} \eta(b, a)\right)^{2}}\right)^{\frac{p}{p-1}}\left(\frac{2^{\alpha}}{2^{\alpha}-1}\right)^{\frac{1}{p-1}} \mid \frac{f(a)+f\left(a+e^{i \varphi} \eta(b, a)\right)}{2} \\
& -\left.\frac{\Gamma(\alpha+1)}{2\left(e^{i \varphi} \eta(b, a)\right)^{\alpha}}\left[J_{a^{+}}^{\alpha} f\left(a+e^{i \varphi} \eta(b, a)\right)+J_{\left(a+e^{i \varphi} \eta(b, a)\right)}^{\alpha} f(a)\right]\right|^{\frac{p}{p-1}} \\
& -\left[\frac{\pi}{2}-\beta\left(\frac{1}{2}, \alpha+\frac{5}{2}\right)-\beta\left(\frac{3}{2}, \alpha+\frac{3}{2}\right)\right]\left(\left|f^{\prime \prime}(a)\right|^{\frac{p-1}{p}}+\frac{1-\lambda}{\lambda}\left|f^{\prime \prime}(b)\right|^{\frac{p-1}{p}}\right) .
\end{aligned}
$$


This leads to

$$
\begin{aligned}
& \left|\frac{f(a)+f\left(a+e^{i \varphi} \eta(b, a)\right)}{2}-\frac{\Gamma(\alpha+1)}{2\left(e^{i \varphi} \eta(b, a)\right)^{\alpha}}\left[J_{a^{+}}^{\alpha} f\left(a+e^{i \varphi} \eta(b, a)\right)+J_{\left(a+e^{i \varphi} \eta(b, a)\right)^{-}}^{\alpha} f(a)\right]\right| \\
& \leq\left(\frac{\left(e^{i \varphi} \eta(b, a)\right)^{2}}{2(\alpha+1)}\right)\left(\frac{2^{\alpha}-1}{2^{\alpha}}\right)^{\frac{1}{p}} \\
& \quad \times\left(\left[\frac{\pi}{2}-\beta\left(\frac{1}{2}, \alpha+\frac{5}{2}\right)-\beta\left(\frac{3}{2}, \alpha+\frac{3}{2}\right)\right]\left(\left|f^{\prime \prime}(a)\right|^{\frac{p}{p-1}}+\frac{1-\lambda}{\lambda}\left|f^{\prime \prime}(b)\right|^{\frac{p}{p-1}}\right)\right)^{\frac{p-1}{p}} .
\end{aligned}
$$

This ends the proof.

Remark 19 In Corollary 17, if we choose

(a) $\eta(b, a)=b-a$ and $\varphi=0$, we get

$$
\begin{aligned}
& \left|\frac{f(a)+f(b)}{2}-\frac{\Gamma(\alpha+1))}{2(b-a)^{\alpha}}\left[J_{a^{+}}^{\alpha} f(b)+J_{b^{-}}^{\alpha} f(a)\right]\right| \\
& \leq\left(\frac{(b-a)^{2}}{2(\alpha+1)}\right)\left(\frac{2^{\alpha}-1}{2^{\alpha}}\right)^{\frac{1}{p}}\left(\left[\frac{\pi}{2}-\beta\left(\frac{1}{2}, \alpha+\frac{5}{2}\right)-\beta\left(\frac{3}{2}, \alpha+\frac{3}{2}\right)\right]\right. \\
& \left.\quad \times\left(\left|f^{\prime \prime}(a)\right|^{\frac{p}{p-1}}+\frac{1-\lambda}{\lambda}\left|f^{\prime \prime}(b)\right|^{\frac{p}{p-1}}\right)\right)^{\frac{p-1}{p}} .
\end{aligned}
$$

(b) $\eta(b, a)=b-a, \varphi=0$, and $\lambda=\frac{1}{2}$, we get

$$
\begin{aligned}
& \left|\frac{f(a)+f(b)}{2}-\frac{\Gamma(\alpha+1))}{2(b-a)^{\alpha}}\left[J_{a^{+}}^{\alpha} f(b)+J_{b^{-}}^{\alpha} f(a)\right]\right| \\
& \leq\left(\frac{(b-a)^{2}}{2(\alpha+1)}\right)\left(\frac{2^{\alpha}-1}{2^{\alpha}}\right)^{\frac{1}{p}}\left(\left[\frac{\pi}{2}-\beta\left(\frac{1}{2}, \alpha+\frac{5}{2}\right)-\beta\left(\frac{3}{2}, \alpha+\frac{3}{2}\right)\right]\right. \\
& \left.\quad \times\left(\left|f^{\prime \prime}(a)\right|^{\frac{p}{p-1}}+\left|f^{\prime \prime}(b)\right|^{\frac{p}{p-1}}\right)\right)^{\frac{p-1}{p}} .
\end{aligned}
$$

Corollary 18 Under the assumptions of Theorem 14. If $\left|f^{\prime \prime}\right|^{\frac{p}{p-1}}$ is h-convex, we have

$$
\begin{aligned}
& \left|\frac{f(a)+f\left(a+e^{i \varphi} \eta(b, a)\right)}{2}-\frac{\Gamma(\alpha+1))}{2\left(e^{i \varphi} \eta(b, a)\right)^{\alpha}}\left[J_{a^{+}}^{\alpha} f\left(a+e^{i \varphi} \eta(b, a)\right)+J_{\left(a+e^{i \varphi} \eta(b, a)\right)^{-}}^{\alpha} f(a)\right]\right| \\
& \leq\left(\frac{\left(e^{i \varphi} \eta(b, a)\right)^{2}}{2(\alpha+1)}\right)\left(\frac{2^{\alpha}-1}{2^{\alpha}}\right)^{\frac{1}{p}} \\
& \quad \times\left(\int_{0}^{1} h(t)\left|(1-t)^{\alpha}-t^{\alpha}\right| d t\right)^{\frac{p-1}{p}}\left(\left|f^{\prime}(a)\right|^{\frac{p}{p-1}}+\frac{1-\lambda}{\lambda}\left|f^{\prime}(b)\right|^{\frac{p}{p-1}}\right)^{\frac{p-1}{p}} .
\end{aligned}
$$

Proof Using (9), by Theorem 14, it can be proved easily. It is omitted.

\section{Conclusion}

In the present paper, using the notion of $F$ and $F$-convex function (see [17]), we construct some new inequalities of Hermite-Hadamard type for differentiable function via 
Riemann-Liouville fractional integral. We also established some trapezoid type inequalities for a function of whose second derivatives absolutely values are $F$-convex. Moreover, we obtained some new inequalities of Hermite-Hadamard type for Riemann-Liouville fractional integrals and via classical integrals. The results presented in this paper would provide generalizations and extension of those given in earlier work.

\section{Acknowledgements}

The authors would like to express their thanks to the editor and the referees for their helpful comments.

Funding

Not applicable.

Availability of data and materials

Not applicable.

\section{Competing interests}

The authors declare that they have no competing interests.

Consent for publication

Not applicable.

Authors' contributions

All authors contributed equally and significantly in writing this article. All authors read and approved the final manuscript.

\section{Author details}

'Department of Mathematics, College of Education, University of Sulaimani, Sulaimani, Iraq. ${ }^{2}$ Department of Mathematics, Faculty of Science and Arts, Düzce University, Düzce, Turkey.

\section{Publisher's Note}

Springer Nature remains neutral with regard to jurisdictional claims in published maps and institutional affiliations.

Received: 31 October 2018 Accepted: 18 December 2018 Published online: 29 December 2018

\section{References}

1. Niculescu, C., Persson, L.E.: Convex Functions and Their Application. Springer, Berlin (2004)

2. Mohammed, P.O.: Inequalities of type Hermite-Hadamard for fractional integrals via differentiable convex functions. Turk. J. Anal. Number Theory 4(5), 135-139 (2016)

3. Alomari, M., Darus, M., Kirmaci, U.S.: Refinements of Hadamard-type inequalities for quasi-convex functions with applications to trapezoidal formula and to special means. Comput. Math. Appl. 59, 225-232 (2010)

4. Noor, M.A., Noor, K.I., Awan, M.U.: Some quantum estimates for Hermite-Hadamard inequalities. Appl. Math. Comput. 251, 675-679 (2015)

5. İşcana, I., Turhan, S.: Generalized Hermite-Hadamard-Fejer type inequalities for GA-convex functions via fractional integral. Moroccan J. Pure Appl. Anal. 2(1), 34-46 (2016)

6. Wu, Y., Qi, F.: On some Hermite-Hadamard type inequalities for (s, QC)-convex functions. SpringerPlus 5, 49 (2016). https://doi.org/10.1186/s40064-016-1676-9

7. Mohammed, P.O.: On new trapezoid type inequalities for $h$-convex functions via generalized fractional integral. Fract. Differ. Calc. 6(4), 125-128 (2018)

8. Khan, M.A., Begum, S., Khurshid, Y., Chu, Y.-M.: Ostrowski type inequalities involving conformable fractional integrals. J. Inequal. Appl. 2018, 70 (2018)

9. Defnetti, B.: Sulla strati cazioni convesse. Ann. Mat. Pura Appl. 30, 173-183 (1949)

10. Mangasarian, O.L.: Pseudo-convex functions. SIAM J. Control 3, 281-290 (1965)

11. Mohammed, P.O.: Some new Hermite-Hadamard type inequalities for MT-convex functions on differentiable coordinates. J. King Saud Univ., Sci. 30, 258-262 (2018)

12. Polyak, B.T.: Existence theorems and convergence of minimizing sequences in extremum problems with restrictions. Sov. Math. Dokl. 7, 72-75 (1966)

13. Hyers, D.H., Ulam, S.M.: Approximately convex functions. Proc. Am. Math. Soc. 3, 821-828 (1952)

14. Hudzik, H., Maligranda, L: Some remarks on s-convex functions. Aequ. Math. 48, 100-111 (1994)

15. Varosanec, S.: On h-convexity. J. Math. Anal. Appl. 326(1), 303-311 (2007)

16. Ermeydan, S., Yildirim, H.: Riemann-Liouville fractional Hermite-Hadamard inequalities for differentiable $\lambda_{\varphi}$-preinvex functions. Malaya J. Mat. 4(3), 430-437 (2016)

17. Samet, B.: On an implicit convexity concept and some integral inequalities. J. Inequal. Appl. 2016, 308 (2016)

18. Sarikaya, M.Z., Set, E., Yaldiz, H., Basak, N.: Hermite-Hadamard's inequalities for fractional integrals and related fractional inequalities. Math. Comput. Model. 57, 2403-2407 (2013). https://doi.org/10.1016/j.mcm.2011.12.048

19. Budak, H., Sarikaya, M.Z.: On Ostrowski type inequalities for F-convex function. AIP Conf. Proc. 1833, 020057 (2017). https://doi.org/10.1063/1.4981705

20. Kilbas, A.A., Srivastava, H.M., Trujillo, J.J.: Theory and Applications of Fractional Differential Equations. Elsevier, Amsterdam (2006) 
21. Budak, H., Sarikaya, M.Z., Yildiz, M.K.: Hermite-Hadamard type inequalities for F-convex function involving fractional integrals. Filomat (in press)

22. Deng, J., Wang, J.: Fractional Hermite-Hadamard inequalities for $(\alpha, m)$-logarithmically convex functions. J. Inequal. Appl. 2013, Article ID 364 (2013)

Submit your manuscript to a SpringerOpen ${ }^{\circ}$ journal and benefit from:

- Convenient online submission

$\checkmark$ Rigorous peer review

- Open access: articles freely available online

- High visibility within the field

- Retaining the copyright to your article

Submit your next manuscript at $\boldsymbol{s p r i n g e r o p e n . c o m ~}$ 